\title{
Die neuen Begleitgesetze zum Vertrag von Lissabon aus Sicht des Deutschen Bundestages - offene Fragen und neue Herausforderungen
}

\author{
Sebastian Gröning-von Thüna*
}

Mit seinem Urteil vom 30. Juni $2009^{1}$ bestätigte das Bundesverfassungsgericht die Vereinbarkeit des Vertrages von Lissabon mit dem Grundgesetz (GG). Zugleich erklärte es das Begleitgesetz ${ }^{2} \mathrm{zu}$ dem Vertrag für verfassungswidrig, da die Beteiligungsrechte von Bundestag und Bundesrat hierin nicht in dem gebotenen Umfang gesichert seien. ${ }^{3}$

Mit den neuen Begleitgesetzen, ${ }^{4}$ die seit dem 25. September 2009 in Kraft sind, behoben Bundestag und Bundesrat diese Mängel und ermöglichten somit die Ratifikation des Vertrages durch Deutschland. Das Bundesverfassungsgericht hatte in Ziffer 4a seines Urteils ${ }^{5}$ eine entsprechende gesetzliche Ausgestaltung der Beteiligungsrechte zur Voraussetzung für die Ratifikation gemacht, die mit der Hinterlegung der Ratifikationsurkunde in Rom noch am Tag des Inkrafttretens der neuen Begleitgesetze abgeschlossen wurde.

Die neuen Begleitgesetze erfüllen aber nicht nur die unmittelbaren Vorgaben des Bundesverfassungsgerichts, die Voraussetzung für die Ratifikation des Vertrages von Lissabon durch Deutschland waren. Bundestag und Bundesrat haben darüber hinaus mit der Änderung der Zusammenarbeitsgesetze, dem Gesetz über die Zusammenarbeit von Bundesregierung und Bundestag (EUZBBG) ${ }^{6}$ und dem Gesetz über die Zusammenarbeit von Bundesregierung und Bundesländern (EUZBLG), ${ }^{7}$ ihre Mitwirkungsrechte in der Europapolitik gestärkt und somit die Grundlage für eine stärkere Rolle im europapolitischen Alltagsgeschäft geschaffen.

* Sebastian Gröning-von Thüna, Büroleiter der Ministerin für Bundesangelegenheiten, Europa und Medien des Landes Nordrhein-Westfalen, Dr. Angelica Schwall-Düren, Düsseldorf. Der Autor war bis August 2010 europapolitischer Referent der SPD-Bundestagsfraktion. Der Artikel gibt ausschließlich die persönliche Auffassung des Autors wieder.

1 BVerfG: Verbundene Rechtssachen 2 BvE 2/08, 2 BvE 5/08, 2 BvR 1010/08, 2 BvR 1022/08, 2 BvR 1259/08, 2 BvR 182/09 vom 30. Juni 2009.

2 Gesetzentwurf der Fraktionen CDU/CSU, SPD und Bündnis 90/Die Grünen. Entwurf eines Gesetzes über die Ausweitung und Stärkung der Rechte des Bundestages und des Bundesrates in Angelegenheiten der Europäischen Union, Bundestagsdrucksache 16/8489 vom 11. März 2008. Das Begleitgesetz von 2008 wurde von Bundestag und Bundesrat beschlossen, aufgrund der Klagen vor dem Bundesverfassungsgericht aber vom Bundespräsidenten nicht unterzeichnet, weshalb nur der Gesetzentwurf existiert.

3 BVerfG: 2 BvE 2/08, 2009, Rn. 406.

4 Gesetz über die Ausweitung und Stärkung der Rechte des Bundestages und des Bundesrates in Angelegenheiten der Europäischen Union vom 22. September 2009 (BGB1. I 2009, S. 3022-3025); Gesetz zur Änderung des Gesetzes über die Zusammenarbeit von Bundesregierung und Deutschem Bundestag in Angelegenheiten der Europäischen Union vom 22. September 2009 (BGB1. I 2009, S. 3026-3030); Gesetz zur Änderung des Gesetzes über die Zusammenarbeit von Bund und Ländern in Angelegenheiten der Europäischen Union vom 22. September 2009 (BGB1. I 2009, S. 3031-3035).

5 BVerfG: 2 BvE 2/08, 2009, Ziffer 4a.

6 Gesetz über die Zusammenarbeit von Bundesregierung und Deutschem Bundestag in Angelegenheiten der Europäischen Union (EUZBBG) vom 12. März 1993 (BGB1. 1993 I, S. 311-312) zuletzt geändert durch Gesetz vom 22. September 2009 (BGB1. I 2009, S. 3026-3030).

7 Gesetz über die Zusammenarbeit von Bundesregierung und Bundesländern in Angelegenheiten der Europäischen Union (EUZBLG) vom 12. März 1993 (BGBl. 1993 I, S. 313-315) zuletzt geändert durch Gesetz vom 22. September 2009 (BGB1. I 2009, S. 3031-3035). 
Ein Jahr nach ihrem Inkrafttreten ergibt die Umsetzung der neuen Begleitgesetze eine gemischte Bilanz. Während durchaus ein verstärktes europapolitisches Engagement erkennbar ist, bleibt die Frage offen, ob dies eine direkte Folge der neuen Rechte und Pflichten aus den Begleitgesetzen ist oder ob die Ursache nicht vielmehr in einem Bewusstseinswandel liegt, der infolge des Urteils und der Verabschiedung der neuen Begleitgesetze stattgefunden hat. Zugleich hat die Praxis sowohl gesetzestechnische als auch politische Schwachpunkte der Begleitgesetze offenbart, die schon jetzt Anlass für eine Diskussion über ihre Überarbeitung bieten.

Hierzu gehören nicht zuletzt die in der Eurokrise offenbar gewordenen Grenzen der Parlamentsbeteiligung bei kurzfristigen Beschlüssen des Europäischen Rates. Entgegen den Bemühungen des Bundestages um eine Stärkung der parlamentarischen Mitbestimmung mussten die Abgeordneten im Frühjahr 2010 gleich zweimal erleben, dass sie Entscheidungen des Europäischen Rates mit erheblicher finanzieller Bedeutung für den Bund in Form der Finanzhilfen für Griechenland ${ }^{8}$ und der Stabilisierung des Euros ${ }^{9}$ nur noch abnicken oder ablehnen, nicht aber mitgestalten konnten. ${ }^{10}$ Dabei stehen sich die Sicherstellung der Handlungsfähigkeit der Europäischen Union in Notfällen einerseits und die notwendige demokratische Legitimierung andererseits anscheinend diametral entgegen.

Neben Forderungen nach neuen ,Eilverfahren' für die Parlamentsbeteiligung in derartigen Notfällen (siehe unten) wirft die Erfahrung mit der Eurokrise die Frage nach der Rolle von Bundestag und Bundesrat in einer sich durch den Vertrag von Lissabon verändernden Machtstruktur in der Europäischen Union auf. Dabei tritt an die Stelle des klassischen Machtkampfes zwischen Kommission, Rat und Parlament eine zunehmende Präsidialisierung, die sich in der Stärkung des Europäischen Rates gegenüber den Fachministerräten äußert. ${ }^{11}$ Diese stärkt innerstaatlich die Staats- und Regierungschefs gegenüber den Fachministern beziehungsweise auf europäischer Ebene den Kommissionspräsidenten gegenüber den Kommissionsmitgliedern.

Ziel dieses Aufsatzes ist, einen Überblick der wichtigsten Erfahrungen und Herausforderungen bei der Umsetzung und Anwendung der neuen Begleitgesetze aus der Perspektive des Bundestages zu geben und mögliche Antworten auf bestehende Schwachstellen aufzuweisen.

\section{Integrationsverantwortung und Mitwirkungsrechte}

Das Bundesverfassungsgericht hat im Urteil über den Vertrag von Lissabon systematisch auf dem Maastricht-Urteil von $1992^{12}$ aufgebaut und den Grundsatz der beschränkten Einzelermächtigung in den Mittelpunkt seiner Bewertung des Vertrages von Lissabon gestellt. Die Frage nach der Vereinbarkeit des Vertrages von Lissabon mit dem GG, entscheidet sich demnach nicht an den Mitwirkungsmöglichkeiten von Bundestag und Bundesrat in der Eu-

8 Gesetz zur Übernahme von Gewährleistungen zum Erhalt der für die Finanzstabilität in der Währungsunion erforderlichen Zahlungsfähigkeit der Hellenischen Republik (Währungsunion-Finanzstabilitätsgesetz - WFStG) (BGB1. 2010 I, S. 537-538).

9 Gesetz zur Übernahme von Gewährleistungen im Rahmen eines europäischen Stabilisierungsmechanismus (BGB1. 2010 I, S. 627-628).

10 Der Ärger über dieses Verfahren wurde insbesondere in den Redebeiträgen der Opposition in der Plenardebatte anlässlich der Entscheidung über das Euro-Rettungspaket vom 21. Mai 2010 zum Ausdruck gebracht. Vgl. Deutscher Bundestag. Stenografischer Bericht. 44. Sitzung. Berlin, Freitag, den 21. Mai 2010, Plenarprotokoll 17/44, S. 4412 (C)-4445 (D).

11 Zur neuen Machtbalance in der Europäischen Union siehe auch Wolfgang Wessel/Thomas Traguth: Der hauptamtliche Präsident des Europäischen Rates: ,Herr“ oder ,Diener“ im Haus Europa, in diesem Band der integration, S. 297-311.

12 BVerfGE 89, 155. 
ropapolitik der Bundesregierung im Sinne der Absätze 2 und 3 des Artikels 23 GG sondern an ihrer Beteiligung bei der Veränderung des Vertragsrechts im Rahmen von Artikel $23 \mathrm{Ab}-$ satz 1 des GG. ${ }^{13}$ Gegenstand des Richterspruches sind also nicht die Mitwirkungsmöglichkeiten von Bundestag und Bundesrat an Rechtsetzungsakten der Europäischen Union, sondern die Frage, ob der Grundsatz der begrenzten Einzelermächtigung durch die Ratifizierung des Vertrages und durch die Begleitgesetze eingehalten wird.

Dies war nicht der Fall. Der Vertrag von Lissabon enthält eine ganze Reihe von Möglichkeiten der ,dynamischen Vertragsentwicklung' wie zum Beispiel die Brückenklausel des Artikels 81 Absatz 3 AEUV $^{14}$ im Bereich des grenzüberschreitenden Familienrechts. Grundsätzlich schreibt Artikel 81 Absatz 3 Unterabsatz 1 AEUV vor, dass Maßnahmen zum Familienrecht mit grenzüberschreitendem Bezug vom Rat einstimmig nach Anhörung des Europäischen Parlaments beschlossen werden. Die Brückenklausel in Artikel 81 Absatz 3 Unterabsatz 2 AEUV ermöglicht dem Rat, einen Beschluss zu erlassen, durch den bestimmte Aspekte des Familienrechts mit grenzüberschreitendem Bezug in das ordentliche Gesetzgebungsverfahren überführt werden, womit es zu Mehrheitsentscheidungen bei gleichberechtigter Mitentscheidung durch das Europäische Parlament kommt. Solche Möglichkeiten der dynamischen Vertragsentwicklung erlauben eine Weiterentwicklung des vertragsrechtlichen Rahmens ohne Ratifikation in allen Mitgliedstaaten und in vereinfachten Verfahren. Angesichts der langwierigen Verhandlungen über vorausgegangene ordentliche Vertragsänderungen sowie der Probleme bei ihrer Ratifikation erscheint die mit den dynamischen Vertragsentwicklungsmöglichkeiten geschaffene Flexibilität durchaus sinnvoll. Für den damit möglichen Fall der Änderungen des Vertragsrechts ohne Ratifikationsverfahren stellt das Bundesverfassungsgericht in seinen Leitsätzen für die Bundesregierung und die gesetzgebenden Körperschaften (Bundestag und Bundesrat), ,eine besondere Verantwortung im Rahmen der Mitwirkung [fest], die in Deutschland innerstaatlich den Anforderungen des Art. 23 Abs. 1 GG genügen muss (Integrationsverantwortung) und gegebenenfalls in einem verfassungsgerichtlichen Verfahren eingefordert werden kann." 15 Da mit der Anwendung von solchen Vertragsentwicklungsklauseln der vertragsrechtliche Rahmen über die im Ratifikationsbeschluss enthaltene begrenzte Einzelermächtigung hinaus geändert werde, fordert das Bundesverfassungsgericht als Voraussetzung für die Zustimmung der Bundesregierung zu derartigen Beschlüssen auf Ebene der Europäischen Union eine entsprechende gesetzliche Ermächtigung.

Das ursprüngliche Begleitgesetz ${ }^{16}$ sah für die Anwendung der dynamischen Vertragsentwicklung keinerlei gesonderte Beteiligung der gesetzgebenden Körperschaften vor. Allerdings verwies der Bundestag in seiner Entschließung zu dem Zustimmungsgesetz darauf, dass das Abstimmungsverhalten der Bundesregierung im Rat beziehungsweise im Europäischen Rat bei der Anwendung der entsprechenden Vertragsentwicklungsmöglichkeiten einer besonderen Rückanbindung an den Deutschen Bundestag bedürfe. Das Begleitgesetz enthielt deshalb eine Aufforderung an die Bundesregierung, den Deutschen Bundestag über anstehende Entscheidungen über die Anwendung der entsprechenden Entwicklungsklauseln sowie über ihre Willensbildung zu informieren, und sich vor der abschließenden Entscheidung im Rat beziehungsweise im Europäischen Rat aktiv um Einvernehmen mit dem Deut-

13 BVerfG: 2 BvE 2/08, 2009, Rn. 410.

14 Vertrag über die Arbeitsweise der Europäischen Union (AEUV), in: Amtsblatt der EU, Nr. C 83 vom 30. März 2010, S. 47-199.

15 BVerfG: 2 BvE 2/08, 2009, Leitsatz 2a.

16 Gesetzentwurf der Fraktionen CDU/CSU, SPD und Bündnis 90/Die Grünen. Entwurf eines Gesetzes über die Ausweitung und Stärkung der Rechte, 2008. 
schen Bundestag zu bemühen. ${ }^{17}$ Für das Verfahren wurde hierbei auf Ziffer VI der Vereinbarung zwischen Bundesregierung und Bundestag über die Zusammenarbeit in Angelegenheiten der Europäischen Union (EUZBBV) ${ }^{18}$ verwiesen, in der die Mitwirkungsrechte des Bundestages bei Beschlüssen über die Aufnahme von Beitritts- und Vertragsverhandlungen geregelt sind.

Diese Vereinbarung betrachtete das Bundesverfassungsgericht jedoch als ungeeignet, um die Wahrnehmung der Integrationsverantwortung von Bundestag und Bundesrat zu gewährleisten. Sie sei ,weder ihrer nicht eindeutigen Rechtsnatur [...] noch ihrem Inhalt nach [...] ausreichend“, um „,die verfassungsrechtlich gebotenen Beteiligungsrechte der gesetzgebenden Körperschaften am europäischen Integrationsprozess im nationalen Recht auf der Ebene des einfachen Gesetzes abzubilden und zu konkretisieren. " ${ }^{19}$ Diese kritische Bewertung der EUZBBV durch das Bundesverfassungsgericht bestätigte die im Parlament bereits vorgebrachte Kritik ${ }^{20}$ an der teils schleppenden Umsetzung sowie einigen Unklarheiten der Zusammenarbeitsgesetze und -vereinbarungen und bot einen Anlass, diese ebenfalls gesetzlich neu zu fassen.

Das Bundesverfassungsgericht verwarf mit seinem Urteil21 im Übrigen auch eine Regelung für das Ablehnungsrecht von Bundestag und Bundesrat bei Anwendung der Brückenklauseln gemäß Artikel 48 Absatz 7 Unterabsatz 3 EUV 22 und gemäß Artikel 81 Absatz 3 Unterabsatz 3 AEUV in Artikel $1 \S 4$ des ursprünglichen Begleitgesetzes. ${ }^{23}$

Die Neufassung der Begleitgesetze teilte sich insofern in ,Pflicht' und ,Kür' auf. Voraussetzung für die Ratifizierung war lediglich die gesetzliche Regelung des Zustimmungserfordernisses im Sinne von Artikel 23 Absatz 1 in Verbindung mit Artikel 79 Absätze 2 und 3 GG bei all jenen Entscheidungen auf europäischer Ebene, mit denen der bestehende Kompetenzrahmen der Europäischen Union ausgeweitet würde (dynamische Vertragsentwicklung). Ferner stellte das Bundesverfassungsgericht auch Änderungsbedarf zur Stärkung des Bundestages bei der Anwendung des Notbremsemechanismus ${ }^{24}$ und des Ablehnungsrechtes gemäß Artikel 48 Absatz 7 Unterabsatz 3 EUV fest. Dieser ,Pflicht-Teil` ist Gegenstand des Integrationsverantwortungsgesetzes (IntVG). ${ }^{25}$ Die Bereiche für die das Primärrecht solche

17 Beschlussempfehlung und Bericht des Ausschusses für die Angelegenheiten der Europäischen Union (21. Ausschuss) zu dem Gesetzentwurf der Bundesregierung - Drucksache 16/8300 - Entwurf eines Gesetzes zum Vertrag von Lissabon vom 13. Dezember 2007, Bundestagsdrucksache 16/8917 vom 23. April 2008, S. 5.

18 Vereinbarung zwischen dem Deutschen Bundestag und der Bundesregierung über die Zusammenarbeit in Angelegenheiten der Europäischen Union vom 30. September 2006 (BGB1. 2006 I, S. 2177-2180).

19 BVerfG: 2 BvE 2/08, 2009, Rn. 410.

20 Die Kritik wurde in den Anträgen der Fraktion Bündnis 90/Die Grünen und der Regierungskoalition sowie in den Plenardebatten zu diesen Anträgen am 5. März und 28. Mai 2009 in unterschiedlicher Deutlichkeit zum Ausdruck gebracht. Vgl. Antrag der Abgeordneten Rainder Steenblock u.a. und der Fraktion Bündnis 90/Die Grünen. Zwei Jahre Europa-Vereinbarung - Bundesregierung muss ihre Verpflichtungen unverzüglich vollständig erfüllen, Bundestagsdrucksache 16/12109 vom 4. März 2009; Antrag der Fraktionen der CDU/CSU, SPD und FDP. Vereinbarung über Zusammenarbeit in Angelegenheiten der Europäischen Union ist einzigartig in Europa - Auslegungsfragen müssen geklärt, noch bestehende Defizite beseitigt werden, Bundestagsdrucksache 16/13169 vom 27. Mai 2009; Deutscher Bundestag. Stenografischer Bericht. 208. Sitzung. Berlin, Donnerstag, den 5. März 2009, Plenarprotokoll 16/208, S. 22530 (A)-22536 (C); Deutscher Bundestag. Stenografischer Bericht. 224. Sitzung. Berlin, Donnerstag, den 28. Mai 2009, Plenarprotokoll 16/224, S. 24686 (B)-24698 (B).

21 BVerfG: 2 BvE 2/08, 2009, Rn. 415.

22 Vertrag über die Europäische Union in der Fassung des Vertrages von Lissabon, in: Amtsblatt der EU, Nr. C 83 vom 30. März 2010, S. 13-45.

23 Gesetzentwurf der Fraktionen CDU/CSU, SPD und Bündnis 90/Die Grünen. Entwurf eines Gesetzes über die Ausweitung und Stärkung der Rechte, 2008.

24 Artikel 48 Absatz 2, Artikel 82 Absatz 3 und Artikel 83 Absatz 3 AEUV.

25 Gesetz über die Wahrnehmung der Integrationsverantwortung des Bundestages und des Bundesrates in Angelegenheiten der Europäischen Union (Integrationsverantwortungsgesetz - IntVG) vom 22. September (BGB1. I 2009, S. 3022-3024) zuletzt geändert durch Gesetz vom 1. Dezember 2009 (BGB1. 2009 I, S. 3822-3823). 
Vertragsentwicklungsmöglichkeiten bietet und die Form der nach Ansicht des Bundesverfassungsgerichts erforderlichen Parlamentsbeteiligung sind in dem Urteil des Bundesverfassungsgerichts ausführlich aufgeführt. ${ }^{26}$ Die Formulierung und Verhandlung der zur Ratifizierung erforderlichen Neuregelung ergab sich insoweit unmittelbar aus dem Urteil.

Die gesetzliche Neuregelung der Mitwirkungsrechte von Bundestag und Bundesrat bei der politischen Gestaltung der Europäischen Union und bei europäischen Rechtsetzungsakten im Sinne der Absätze 2, 3 und 4 des Artikels 23 GG war für die Ratifizierung nicht erforderlich. Angesichts der Kritik des Bundesverfassungsgerichts ${ }^{27}$ und der Bundestagsfraktio$n^{28}$ an der EUZBBV war die Überführung des Inhaltes der EUZBBV in das EUZBBG jedoch naheliegend. Die gesetzliche Neuregelung bot zugleich eine Option, die bestehenden Mängel und Schwierigkeiten bei der Umsetzung zu beheben. Insbesondere Lücken bei der Information des Bundestages durch die Bundesregierung und Auslegungsdifferenzen bezüglich der Handhabung der Einvernehmensherstellung vor Aufnahme von Verhandlungen über Beitritte oder Vertragsänderungen sollte die neue gesetzliche Regelung überwinden helfen. ${ }^{29}$ Zugleich versprach sich der Bundestag von der gesetzlichen Regelung ein höheres $\mathrm{Maß}$ an Verbindlichkeit, ${ }^{30}$ da das Gesetz immer auch Grundlage einer Organklage sein könnte, die Vereinbarung hingegen nicht. Dieser ,Kür-Teil` ist Gegenstand der beiden Zusammenarbeitsgesetze EUZBBG und EUZBLG.

In der politischen Praxis hat sich die Bedeutung der Begleitgesetze im Verhältnis zu ihrer Relevanz für die Ratifikation umgekehrt. Die Kür wurde zur Pflicht und die Pflicht zur Kür. ${ }^{31}$

26 BVerfG: 2 BvE 2/08, 2009, Rn 411-419.

27 Ebenda, Rn. 410.

28 Vgl. Antrag der Abgeordneten Rainder Steenblock u.a. und der Fraktion Bündnis 90/Die Grünen. Zwei Jahre Europa-Vereinbarung, 2009; Antrag der Fraktionen der CDU/CSU, SPD und FDP. Vereinbarung über Zusammenarbeit, 2009; Deutscher Bundestag. Stenografischer Bericht. 208. Sitzung. Berlin, Donnerstag, den 5. März 2009, Plenarprotokoll 16/208, S. 22530 (A)-22536 (C); Deutscher Bundestag. Stenografischer Bericht. 224. Sitzung. Berlin, Donnerstag, den 28. Mai 2009, Plenarprotokoll 16/224, S. 24686 (B)-24698 (B).

29 Gemäß Ziffer VI Absatz 2 EUZBBV sollte die Bundesregierung sich bei Verhandlungen über Beitritte oder Vertragsänderungen vor der abschließenden Entscheidung im Rat bemühen, Einvernehmen mit dem Bundestag herzustellen. Die Bundesregierung vertrat die Auffassung, dass die formale Bitte um die Herstellung des Einvernehmens nur dann notwendig sei, wenn der Bundestag zuvor eine Stellungnahme zu der jeweiligen Unterrichtung der Bundesregierung über die Aufnahme der entsprechenden Verhandlungen abgegeben hatte und ein Dissens zwischen dieser Stellungnahme und der Verhandlungsposition der Bundesregierung bestand. Im Bundestag wurde hingegen mehrheitlich die Auffassung vertreten, dass eine solche formale Bitte um die Herstellung des Einvernehmens auf jeden Fall erfolgen müsse, unabhängig davon, ob zuvor eine Stellungnahme abgegeben worden sei.

30 Siehe Redebeitrag des Vorsitzenden des Ausschusses für die Angelegenheiten der Europäischen Union Gunther Krichbaum, MdB, zu Tagesordnungspunkt 2 in der Plenardebatte vom 8. September 2009. Vgl. Deutscher Bundestag. Stenografischer Bericht. 233. Sitzung. Berlin, Dienstag, den 8. September 2009, Plenarprotokoll 16/233, S. 26349 (D)-26351 (A).

31 Siehe hierzu den Abschnitt zu den „Auslegungsschwierigkeiten bei der Herstellung des Einvernehmens“ weiter unten. 
Tabelle: Synopse der verfassungsrechtlich relevanten Begleitregelungen zum Vertrag von Lissabon

\begin{tabular}{|c|c|c|c|c|c|}
\hline & \multicolumn{2}{|c|}{ Begleitgesetzentwurf 2008} & \multicolumn{3}{|c|}{ Begleitgesetz 2009} \\
\hline & $\begin{array}{l}\text { Gesetz über } \\
\text { die Ausübung } \\
\text { der Rechte }^{\mathrm{a}}\end{array}$ & $\begin{array}{l}\text { Bezug im } \\
\text { EU-Recht }\end{array}$ & IntGV $^{b}$ & $\begin{array}{l}\text { Beschluss- } \\
\text { form/nat. } \\
\text { Recht }^{\text {c }}\end{array}$ & $\begin{array}{l}\text { Bezug im } \\
\text { EU-Recht }\end{array}$ \\
\hline $\begin{array}{l}\text { Vereinfachtes } \\
\text { Vertragsände- } \\
\text { rungsverfahren }\end{array}$ & & & $\S 2$ & $\begin{array}{l}\text { Art. } 23 \\
\text { Abs. } 1 \text { GG }\end{array}$ & $\begin{array}{l}\text { Art. } 48 \text { Abs. } 6 \text { UAbs. } 2 \\
\text { und } 3 \text { EUV }\end{array}$ \\
\hline \multirow[t]{3}{*}{$\begin{array}{l}\text { Besondere } \\
\text { Vertragsände- } \\
\text { rungsverfahren }\end{array}$} & & & $\S 3$ Abs. 1 & \multirow[t]{3}{*}{$\begin{array}{l}\text { Art. } 23 \\
\text { Abs. } 1 \text { GG }\end{array}$} & $\begin{array}{l}\text { Art. } 218 \text { Abs. } 8 \text { UAbs. } 2 \\
\text { S. } 2 \text { AEUV; } \\
\text { Art. } 311 \text { Abs. } 3 \text { AEUV }\end{array}$ \\
\hline & & & $\S 3$ Abs. 2 & & $\begin{array}{l}\text { Art. } 25 \text { Abs. } 2 \text { AEUV; } \\
\text { Art. } 223 \text { Abs. } 1 \text { UAbs. } 2 \\
\text { AEUV; } \\
\text { Art. } 262 \text { AEUV }\end{array}$ \\
\hline & & & $\S 3$ Abs. 3 & & $\begin{array}{l}\text { Art. } 42 \text { Abs. } 2 \text { UAbs. } 1 \\
\text { und } 2 \text { EUV }\end{array}$ \\
\hline \multirow[t]{2}{*}{ Brückenklausel } & $\S 4$ Abs. 3 & $\begin{array}{l}\text { Art. } 48 \text { Abs. } 7 \\
\text { EUV }\end{array}$ & $\S 4$ Abs. 1 & \multirow[t]{2}{*}{$\begin{array}{l}\text { Art. } 23 \\
\text { Abs. } 1 \text { GG }\end{array}$} & $\begin{array}{l}\text { Art. } 48 \text { Abs. } 7 \text { UAbs. } 1 \\
\text { und } 2 \text { EUV }\end{array}$ \\
\hline & $\S 4$ Abs. 6 & $\begin{array}{l}\text { Art. } 81 \text { Abs. } 3 \\
\text { UAbs. } 2 \\
\text { AEUV }\end{array}$ & $\S 4$ Abs. 2 & & $\begin{array}{l}\text { Art. } 81 \text { Abs. } 3 \text { UAbs. } 2 \\
\text { AEUV }\end{array}$ \\
\hline \multirow{2}{*}{$\begin{array}{l}\text { Zustimmung } \\
\text { im Europä- } \\
\text { ischen Rat und } \\
\text { im Rat bei be- } \\
\text { sonderen Brü- } \\
\text { ckenklauseln }\end{array}$} & & & $\S 5$ Abs. 1 & \multirow[t]{2}{*}{ Beschluss } & $\begin{array}{l}\text { Art. } 31 \text { Abs. } 3 \text { EUV; } \\
\text { Art. } 312 \text { Abs. } 2 \text { UAbs. } 2 \\
\text { AEUV }\end{array}$ \\
\hline & & & $\S 6$ Abs. 1 & & $\begin{array}{l}\text { Art. } 153 \text { Abs. } 2 \text { UAbs. } 4 \\
\text { AEUV; } \\
\text { Art. } 192 \text { Abs. } 2 \text { UAbs. } 2 \\
\text { AEUV; } \\
\text { Art. } 333 \text { Abs. } 1 \text { und } 2 \\
\text { AEUV }\end{array}$ \\
\hline \multirow[t]{2}{*}{$\begin{array}{l}\text { Kompetenz- } \\
\text { erweiterungs- } \\
\text { klausel }\end{array}$} & Entschließung $^{\mathrm{d}}$ & $\begin{array}{l}\text { Art. } 83 \text { Abs. } 1 \\
\text { UAbs. } 3 \\
\text { AEUV }\end{array}$ & $\S 7$ Abs. 1 & \multirow[t]{2}{*}{$\begin{array}{l}\text { Art. } 23 \\
\text { Abs. } 1 \text { GG }\end{array}$} & $\begin{array}{l}\text { Art. } 83 \text { Abs. } 1 \text { UAbs. } 3 \\
\text { AEUV; } \\
\text { Art. } 86 \text { Abs. } 4 \text { AEUV }\end{array}$ \\
\hline & & & $\S 7$ Abs. 2 & & Art. 308 Abs. 3 AEUV \\
\hline $\begin{array}{l}\text { Flexibilitäts- } \\
\text { klausel }\end{array}$ & & & $\S 8$ & $\begin{array}{l}\text { Art. } 23 \\
\text { Abs. } 1 \text { GG }\end{array}$ & Art. 352 AEUV \\
\hline $\begin{array}{l}\text { Notbremse- } \\
\text { mechanismus }\end{array}$ & Entschließung $^{\mathrm{d}}$ & $\begin{array}{l}\text { Art. } 82 \text { Abs. } 3 \\
\text { AEUV; } \\
\text { Art. } 83 \text { Abs. } 3 \\
\text { AEUV }\end{array}$ & $\S 9$ Abs. 1 & Beschluss & $\begin{array}{l}\text { Art. } 48 \text { Abs. } 2 \text { S. } 1 \\
\text { AEUV; } \\
\text { Art. } 82 \text { Abs. } 3 \text { UAbs. } 1 \\
\text { S. } 1 \text { AEUV; } \\
\text { Art. } 83 \text { Abs. } 3 \text { UAbs. } 1 \\
\text { S. } 1 \text { AEUV }\end{array}$ \\
\hline
\end{tabular}




\begin{tabular}{|c|c|c|c|c|c|}
\hline & \multicolumn{2}{|c|}{ Begleitgesetzentwurf 2008} & \multicolumn{3}{|c|}{ Begleitgesetz 2009} \\
\hline & $\begin{array}{l}\text { Gesetz über } \\
\text { die Ausübung } \\
\text { der Rechte }\end{array}$ & $\begin{array}{l}\text { Bezug im } \\
\text { EU-Recht }\end{array}$ & IntGV $^{b}$ & $\begin{array}{l}\text { Beschluss- } \\
\text { form/nat. } \\
\text { Rechtc }^{\text {c }}\end{array}$ & $\begin{array}{l}\text { Bezug im } \\
\text { EU-Recht }\end{array}$ \\
\hline \multirow{2}{*}{$\begin{array}{l}\text { Ablehnungs- } \\
\text { recht bei Brü- } \\
\text { ckenklauseln }\end{array}$} & & & $\S 10$ Abs. 1 & \multirow[t]{2}{*}{ Beschluss } & $\begin{array}{l}\text { Art. } 48 \text { Abs. } 7 \text { UAbs. } 3 \\
\text { EUV }\end{array}$ \\
\hline & & & $\S 10$ Abs. 3 & & $\begin{array}{l}\text { Art. } 81 \text { Abs. } 3 \text { UAbs. } 3 \\
\text { AEUV }\end{array}$ \\
\hline $\begin{array}{l}\text { Subsidiaritäts- } \\
\text { rüge }\end{array}$ & $\S 2$ Abs. 2 & $\begin{array}{l}\text { Art. } 6 \text { Subsi- } \\
\text { diaritätspro- } \\
\text { tokoll }\end{array}$ & $\S 11$ Abs. 1 & $\begin{array}{l}\S 93 \mathrm{c} \\
\text { GO-BT }\end{array}$ & $\begin{array}{l}\text { Art. } 6 \text { Subsidiaritätspro- } \\
\text { tokoll }\end{array}$ \\
\hline \multirow[t]{3}{*}{$\begin{array}{l}\text { Subsidiaritäts- } \\
\text { klage }\end{array}$} & $\S 3$ Abs. 2 & $\begin{array}{l}\text { Art. } 8 \text { Subsi- } \\
\text { diaritätspro- } \\
\text { tokoll }\end{array}$ & $\S 12$ Abs. 1 & \multirow{3}{*}{$\begin{array}{l}\text { Art. } 23 \\
\text { Abs. 1a GG; } \\
\S 93 \mathrm{~d} \\
\text { GO-BT }\end{array}$} & $\begin{array}{l}\text { Art. } 8 \text { Subsidiaritätspro- } \\
\text { tokoll }\end{array}$ \\
\hline & $\S 3$ Abs. 5 & $\begin{array}{l}\text { Art. } 8 \text { Subsi- } \\
\text { diaritätspro- } \\
\text { tokoll }\end{array}$ & $\S 12$ Abs. 4 & & $\begin{array}{l}\text { Art. } 8 \text { Subsidiaritätspro- } \\
\text { tokoll }\end{array}$ \\
\hline & § 3 Abs. 6 & $\begin{array}{l}\text { Art. } 8 \text { Subsi- } \\
\text { diaritätspro- } \\
\text { tokoll }\end{array}$ & $\S 12$ Abs. 5 & & $\begin{array}{l}\text { Art. } 8 \text { Subsidiaritätspro- } \\
\text { tokoll }\end{array}$ \\
\hline \multirow[t]{4}{*}{ Unterrichtung } & $\S 6$ & & $\S 13$ Abs. 1 & \multirow[t]{4}{*}{ I } & $\begin{array}{l}\text { [Angelegenheiten des } \\
\text { IntVG] }\end{array}$ \\
\hline & $\begin{array}{l}\S 4 \text { Abs. } 1 \text { und } \\
2 \text { (Brücken- } \\
\text { klausel) }\end{array}$ & $\begin{array}{l}\text { Art. } 48 \text { Abs. } 7 \\
\text { EUV }\end{array}$ & $\S 13$ Abs. 2 & & $\begin{array}{l}\text { Art. } 48 \text { Abs. } 7 \text { EUV; } \\
\text { Art. } 81 \text { Abs. } 3 \text { UAbs. } 2 \\
\text { AEUV }\end{array}$ \\
\hline & & & $\S 13$ Abs. 3 & & $\begin{array}{l}\text { Art. } 48 \text { Abs. } 1 \text { AEUV; } \\
\text { Art. } 48 \text { Abs. } 7 \text { EUV; } \\
\text { Art. } 81 \text { Abs. } 3 \text { UAbs. } 2 \\
\text { AEUV; } \\
\text { Art. } 82 \text { Abs. } 2 \text { AEUV; } \\
\text { Art. } 83 \text { Abs. } 1 \text { und } 2 \\
\text { AEUV }\end{array}$ \\
\hline & & & $\S 13$ Abs. 5 & & $\begin{array}{l}\text { Art. } 48 \text { Abs. } 2 \text { S. } 1 \\
\text { AEUV; } \\
\text { Art. } 82 \text { Abs. } 3 \text { UAbs. } 1 \\
\text { S. } 1 \text { AEUV; } \\
\text { Art. } 83 \text { Abs. } 3 \text { UAbs. } 1 \\
\text { S. } 1 \text { AEUV }\end{array}$ \\
\hline \multicolumn{6}{|c|}{$\begin{array}{l}\text { a) Entwurf eines Gesetzes über die Ausweitung und Stärkung der Rechte des Bundestages und des Bun- } \\
\text { desrates in Angelegenheiten der Europäischen Union, } 2008 \text {. } \\
\text { b) Gesetz über die Wahrnehmung der Integrationsverantwortung des Bundestages und des Bundesrates in } \\
\text { Angelegenheiten der Europäischen Union (Integrationsverantwortungsgesetz - IntVG), } 2009 . \\
\text { c) Bis zum horizontalen Trennstrich wird in der Spalte angegeben in welcher Form der Bundestag jeweils } \\
\text { beschließen muss. Unterhalb der Trennlinie enthält die Spalte Bezüge zu weiteren Regelung des natio- } \\
\text { nalen Rechts. } \\
\text { d) Bericht und Beschlussempfehlung des Europaausschusses, 2008 S. S. }\end{array}$} \\
\hline
\end{tabular}

Quelle: Eigene Darstellung. 


\section{Die Anwendung des Integrationsverantwortungsgesetzes}

\section{Die Beteiligungsrechte im Rahmen der dynamischen Vertragsentwicklung}

Eine Anwendung der diversen Vertragsentwicklungsklauseln, die Gegenstand des IntVG sind, ist bislang ausgeblieben. Schon in der Anhörung des Ausschusses für die Angelegenheiten der Europäischen Union (Europaausschuss) zu den Begleitgesetzen am 26. und 27. August 2009 wurde das Gesetz von dem Sachverständigen Christian Calliess als ,,Sonntagsgesetz" bezeichnet, da es auf besondere Fälle des Vertrages von Lissabon ausgerichtet sei, ${ }^{32}$ also seltene, wenn nicht gar auf einmalige Anwendungsfälle zugeschnitten ist.

Eine Ausnahme hiervon könnte $§ 8$ des IntVG darstellen, der das Erfordernis eines Zustimmungsgesetzes in Anwendungsfällen des Artikels 352 AEUV festlegt. Artikel 352 AEUV entspricht dem Artikel 308 EGV, ${ }^{33}$ der auch als Vertragsabrundungsklausel bezeichnet wird. Der Rat kann demnach auf Vorschlag der Kommission und nach Anhörung des Europäischen Parlaments einstimmig Vorschriften erlassen, die im Rahmen des gemeinsamen Marktes erforderlich erscheinen, um die Ziele der Gemeinschaft zu verwirklichen. Die Dehnbarkeit dieser Zweckbestimmung kann beispielsweise daran verdeutlicht werden, dass Artikel 308 EGV auch als Rechtsgrundlage für die Bereitstellung makrofinanzieller Hilfen für Drittländer herangezogen wurde. ${ }^{34}$

Auch die Nutzung dieser Vertragsabrundungsklausel und die damit verbundene Anwendung des IntVG ist seit dem Inkrafttreten der neuen Begleitgesetze ausgeblieben. Zuletzt wurden Makrofinanzhilfen für Georgien, Serbien, Bosnien-Herzegowina und Armenien durch den Rat Justiz und Inneres auf seiner Tagung vom 30. November und 1. Dezember 2009 noch auf der Grundlage des EGV (also in der Fassung des Vertrages von Nizza) angenommen, auf den $\S 8$ des IntVG keine Anwendung findet. ${ }^{35}$

Für die Zukunft erscheint allerdings eine Anwendung insbesondere von $§ 4$ Absatz 2 (gesetzliche Zustimmungserfordernis bei Beschlüssen zum Übergang zum ordentlichen Gesetzgebungsverfahren im grenzüberschreitenden Familienrecht) sowie gegebenenfalls § 9 (Notbremsemechanismus) und $\S 10$ (Ablehnungsrecht bei Brückenklauseln) des IntVG durchaus möglich, da sie Politikbereiche betreffen, die Teil des Stockholmer Programms zur Schaffung des Raumes der Freiheit, der Sicherheit und des Rechts sind und insofern in absehbarer Zeit Gegenstand von Kommissionsinitiativen werden sollen. ${ }^{36}$

32 Redebeitrag von Christian Calliess in der 90. Ausschusssitzung des Ausschusses für die Angelegenheiten der Europäischen Union. Vgl. Deutscher Bundestag: Öffentliche Anhörung. Protokoll der 90. Sitzung des Ausschusses für die Angelegenheiten der Europäischen Union. Gemeinsame Sitzung mit dem Ausschuss für Europäische Fragen des Bundesrates, 26./27.08.2009, S. 47-48.

33 Vertrag zur Gründung der Europäischen Gemeinschaft (EGV) in der Fassung des Vertrages von Nizza, in: Amtsblatt der EU, Nr. C 321 E vom 29. Dezember 2006, S. 37-331.

34 Der Sachverständige Andreas Maurer schätzte in einem Beitrag bei der Anhörung des Europaausschusses zu den Begleitgesetzen, dass der Artikel 352 AEUV und somit auch § 8 des IntVG jährlich etwa 20- bis 30-mal angewandt werden dürfte. Vgl. Deutscher Bundestag: Protokoll der 90. Sitzung des Europaausschusses, 2009, S. 54.

35 Vgl. Rat der Europäischen Union: Mitteilung an die Presse, 2979. Tagung des Rates Justiz und Inneres, Dok. 16883/1/09 REV 1 (Presse 355).

36 Rat der Europäischen Union: Stockholmer Programm - Ein offenes und sicheres Europa im Dienste und zum Schutz der Bürger, Dok. 17024/09. 


\section{Subsidiaritätsrïge}

Eine Sonderstellung haben die $\S \S 11,12$ und 13 des IntVG, die die Anwendung der Subsidiaritätsrüge, der Subsidiaritätsklage und die notwendige Unterrichtung von Bundestag und Bundesrat zur Wahrnehmung der Rechte und Pflichten aus dem IntVG regeln. ${ }^{37}$

Die Konkretisierung und Substantiierung der Subsidiaritätskontrolle im Vertrag von Lissabon wird immer wieder als wesentlicher Fortschritt im Vertragsrecht bezeichnet. Die hervorgehobene Rolle der nationalen Parlamente bei dieser Kontrolle ist zugleich als Beleg für die Stärkung der nationalen Parlamente durch den Vertrag von Lissabon betrachtet worden. ${ }^{38}$ Die praktische Anwendung sowohl der Subsidiaritätsrüge als auch der Subsidiaritätsklage steht aber weiterhin unter dem Vorbehalt sehr unterschiedlicher Auslegungen und Erwartungen sowie praktischer Hemmnisse.

Schon die formalen Voraussetzungen für die Erteilung einer Rüge auf der Grundlage von Artikel 6 in Verbindung mit Artikel 7 des Subsidiaritätsprotokolls des Vertrages von Lissabon ${ }^{39}$ stellen ein enormes Hindernis für die Anwendung dieses Instruments dar: Das nötige Quorum von einem Drittel beziehungsweise einem Viertel aller nationalen Parlamente ${ }^{40}$ muss innerhalb einer achtwöchigen Frist erreicht werden, was eine schnelle und koordinierte Befassung der nationalen Parlamente voraussetzt. Das Zustandekommen des Quorums führt aber noch nicht zur Ablehnung sondern lediglich zur Überprüfung des Gesetzgebungsvorschlages. Nach der Prüfung kann der Initiator des Entwurfs an seinem Vorschlag festhalten, ihn ändern oder ihn zurückziehen. ${ }^{41}$ Dies gilt auch dann, wenn die Anzahl der begründeten Stellungnahmen, in denen eine Verletzung des Subsidiaritätsprinzips festgestellt wird, die einfache Mehrheit der den nationalen Parlamenten zugewiesenen Stimmen erreicht. ${ }^{42}$ Angesichts der vertragsrechtlichen Verpflichtung zur Einhaltung des Subsidiaritätsprinzips ${ }^{43}$ käme eine Abänderung des Gesetzgebungsvorschlages infolge der Rüge einem schwerwiegenden Schuldeingeständnis gleich, was zugleich einen erheblichen Glaubwürdigkeitsverlust nach sich zöge. Ein solcher Vorgang erscheint deshalb politisch eher unwahrscheinlich.

Die Subsidiaritätsrüge ist somit im besten Falle ein Verzögerungsinstrument, mit einem sehr begrenzten gestalterischen Potenzial. In der Praxis dürfte sich die Rüge angesichts dieser vielen Hindernisse als nur mühsam anwendbares und daher unbefriedigendes Instrument erweisen. Selbst wenn man unterstellt, dass die Rüge eines Parlaments aus einem großen Mitgliedstaat auch unabhängig von rechtlichen Konsequenzen diskursive Wirkung entfalten kann, so bleibt gerade dann die Frage, weshalb ein formal so komplexes Verfahren genutzt werden soll, anstelle eines einfachen Antrags, einer Stellungnahme auf der Grundlage von Artikel 23 Absatz 3 GG oder einer öffentlichen Debatte im Bundestagsplenum oder in den Medien.

Dennoch haben sich die nationalen Parlamentarier und Parlamentarierinnen der 27 EUMitgliedstaaten seit 2006 im Rahmen der Konferenz der Ausschüsse für Gemeinschafts- und

37 Die $\S \S 11$ und 12 IntVG geben weitgehend wortgleich die $\S \S 2$ und 3 des ersten Artikels des ursprünglichen Begleitgesetzes wieder, die darin enthaltenen Unterrichtungspflichten wurden aber abgetrennt und in den neuen § 13 IntVG überführt, der zusätzlich all jene Unterrichtungspflichten enthält, die sich aus den sonstigen Regelungen des IntVG ergeben.

38 Vgl. Bericht und Beschlussempfehlung des Europaausschusses, 2008, Punkt 2.

39 Protokoll (Nr. 2) über die Anwendung der Grundsätze der Subsidiarität und der Verhältnismäßigkeit, in: Amtsblatt der EU, Nr. C 83 vom 30. März 2010, S. 206-209.

40 Ein Viertel bei Gesetzgebungsakten im Bereich der Justiziellen Zusammenarbeit in Strafsachen und der Polizeilichen Zusammenarbeit. Vgl. Artikel 7 Absatz 2 Unterabsatz 1 Subsidiaritätsprotokoll.

41 Artikel 7 Absatz 2 Unterabsatz 2 Subsidiaritätsprotokoll.

42 Artikel 7 Absatz 3 Subsidiaritätsprotokoll.

43 Artikel 5 EUV sowie beziehungsweise i.V.m. Artikel 1, 2 und 5 des Subsidiaritätsprotokolls. 
Europa-Angelegenheiten der Parlamente der Europäischen Union (COSAC) ${ }^{44}$ auf die Subsidiaritätsrüge mit sogenannten Testläufen vorbereitet. Dabei wurden periodisch auf der Grundlage der Arbeits- und Legislativprogramme EU-Vorhaben identifiziert, auf deren Prüfung hinsichtlich der Einhaltung des Subsidiaritätsprinzips sich alle Parlamente einigten. Die COSAC hat die Erfahrungen der verschiedenen Mitgliedstaaten mehrmals ausgewertet und somit die Grundlage für einen gegenseitigen Lernprozess der nationalen Parlamente sowie einen Dialog über die bessere Zusammenarbeit bei der Subsidiaritätskontrolle geschaffen. ${ }^{45}$ Dieser Erfahrungsaustausch und die Zusammenarbeit fanden allerdings auf einer Expertenebene statt und wirkten sich nur indirekt auf das politische Alltagsgeschäft aus. Verbesserungsvorschläge, wie etwa die verstärkte Verabredung von Subsidiaritätskontrollen auf der Grundlage des Arbeits- und Legislativprogramms der Kommission oder die frühzeitige Anzeige von Subsidiaritätsbedenken noch vor der formalen Annahme eines entsprechenden Beschlusses, ${ }^{46}$ sind gut und richtig, wurden im Bundestag aber bislang nicht systematisch umgesetzt.

Dafür gibt es Gründe: So obliegt im Bundestag etwa die Subsidiaritätsprüfung den Fachausschüssen. ${ }^{47}$ Der Europaausschuss ist zwar für die Vertretung des Bundestages in der COSAC zuständig, eine Koordinierungsfunktion für die Subsidiaritätskontrolle innerhalb des Bundestages hat er aber nicht. Von einer solchen Koordinierung kann nur auf Verwaltungsebene gesprochen werden, wo die Unterabteilung Ausschüsse (PA) der Bundestagsverwaltung für die Betreuung aller Ausschüsse und für die Koordinierung der Ausschussarbeiten zuständig ist. Aus der Verwaltung kann die Initiative zur Formulierung einer Subsidiaritätsrüge allerdings nicht erfolgen. Diese Entscheidung ist eine politische und muss dementsprechend von den Abgeordneten gefasst werden.

Der Vorschlag, sich zwischen den nationalen Parlamenten vorab auf eine vertiefte Prüfung bestimmter, von der Kommission in ihrem Arbeits- und Legislativprogramm angekündigter Maßnahmen zu einigen, würde eine solche politische Koordinierung voraussetzen, sowohl nach außen, gegenüber der COSAC, als auch nach innen, zwischen den Ausschüssen. Voraussetzung wäre, dass die Fachausschüsse zunächst Vorschläge für eine vertiefte Prüfung machten und anschließend die (wahrscheinlich im Rahmen der COSAC) vereinbarten Prüfungen durchführten. Dies würde bedeuten, dass die Fachausschüsse sich in ihrer Arbeitsplanung und bei der Gestaltung der Tagesordnung vorab festlegen müssten und sich in-

44 Conférence des Organes Spécialisés dans les Affaires Communautaires et Européennes des Parlements de l'Union européenne (COSAC).

45 Siehe 7. Halbjahresbericht der COSAC sowie den Bericht einer Arbeitsgruppe zur Umsetzung des Protokolls über die Anwendung der Grundsätze der Subsidiarität und Verhältnismäßigkeit. Vgl. COSAC Sekretariat: Seventh bi-annual report: Developments in European Union. Procedures and Practices. Relevant to Parliamentary Scrutiny. Prepared by the COSAC Secretariat and presented to: XXXVII Conference of Community and European Affairs Committees of Parliaments of the European Union. 13-15 May 2007, Brüssel 2007, S. 6-14; Report on the results of the Working Group of the National Parliaments' Representatives to the EU on the Implementation of the Protocol 2 on the Application of the Principles of Subsidiarity and Proportionality as attached to the Treaty of Lisbon, in: COSAC Sekretariat: Tenth Bi-annual Report: Developments in European Union Procedures and Practices Relevant to Parliamentary Scrutiny. Prepared by the COSAC Secretariat and presented to: XL Conference of Community and European Affairs Committees of Parliaments of the European Union. 3-4 November 2008, Brüssel 2008, S. 15-23.

46 Beide Vorschläge wurden von der Arbeitsgruppe der Experten aus den nationalen Parlamenten zur Umsetzung des Protokolls über die Anwendung der Grundsätze der Subsidiarität und Verhältnismäßigkeit im Detail ausgearbeitet und im 10. Halbjahresbericht der COSAC vom November 2008 präsentiert. Vgl. Report on the results of the Working Group of the National Parliaments' Representatives to the EU, 2008, S. 17-20.

$47 \S 93$ a Absatz 1 der Geschäftsordnung des Deutschen Bundestages (GO-BT) in der Fassung der Bekanntmachung vom 2. Juli 1980 (BGB1. I 1980, S. 1237), zuletzt geändert laut Bekanntmachung vom 16. Juli 2010 (BGB1. I 2010, S. 1041). 
sofern Vorgaben machen ließen. Unabhängig von der Frage, ob dies praktisch und politisch machbar wäre, stünde ein solches Vorgehen im Widerspruch zur gängigen Praxis, bei der sich die Tagesordnungen der meisten Bundestagsausschüsse an den Initiativen der Fraktionen und der Bundesregierung sowie an der politischen Aktualität orientieren. Eine solche Festlegung der Tagesordnung aufgrund von mittelfristigen interparlamentarischen Absprachen wäre auch mit Blick auf die Geschäftsordnung des Bundestages fragwürdig. ${ }^{48}$

Der Vorschlag der COSAC-Expertengruppe, die Zusammenarbeit der Parlamente bei der Subsidiaritätskontrolle durch eine frühzeitige Anzeige von Subsidiaritätsbedenken, etwa über das interparlamentarische EU-Informationsaustauschsystem IPEX, ${ }^{49} \mathrm{zu}$ verbessern, scheitert an der politischen Realität, in der eine Ankündigung stets auch eine Festlegung ist. ${ }^{50}$ Die Annahme, ein Parlament könne öffentlich ohne weiterreichende politische Konsequenzen ankündigen, bei einem bestimmten Rechtsetzungsvorhaben der Kommission bestünden eventuell Subsidiaritätsbedenken, erscheint bestenfalls naiv. Unabhängig davon, dass auch hier das Problem der internen Koordinierung besteht (wer entscheidet, dass mögliche Bedenken bestehen, und wer meldet dies an IPEX?), widerspricht es der Arbeitsweise des Parlaments, sich öffentlich zu positionieren, ohne diese Positionierung zuvor sachlich und politisch umfänglich geprüft zu haben. Es entspricht auch nicht der parlamentarischen Praxis, Anträge vor ihrer formalen Einbringung durch die Fraktionen zu veröffentlichen. Üblich ist vielmehr die informelle Vorklärung zwischen Koalitionspartnern oder den Unterstützern eines Antrages vor der formellen Einbringung. Eine solche informelle Zusammenarbeit zwischen den nationalen Parlamenten beziehungsweise zwischen den Abgeordneten ist bislang die Ausnahme, was nicht zuletzt dem dazu notwendigen Aufwand geschuldet sein dürfte. ${ }^{51}$

Eine informelle Vorabklärung ist auch insofern notwendig, da die Bewertung, ob ein Vorschlag das Prinzip der Subsidiarität berücksichtigt oder nicht, eine politische ist und insofern eine Beurteilung auf der Grundlage vorab vereinbarter Prüfschemata kaum realisierbar sein dürfte. Das Ergebnis dürfte vielmehr je nach mitgliedstaatlicher Perspektive und je nach parteipolitischer Prägung unterschiedlich ausfallen. Die Antwort auf die Frage, ob ,die Ziele der in Betracht gezogenen Maßnahmen von den Mitgliedstaaten weder auf zentraler noch auf regionaler oder lokaler Ebene ausreichend verwirklicht werden können“, 52 entscheidet sich maßgeblich an der politischen Grundhaltung.

Hinzu kommt, dass es bezüglich des Umfangs und der Kriterien für die Subsidiaritätsprüfung durchaus unterschiedliche Auffassungen gibt. Insbesondere die Frage, ob die Subsidiaritätsprüfung auch die Prüfung der Kompetenzgrundlage und der Verhältnismäßigkeit umfassen sollte oder ob ausschließlich die Subsidiarität im engeren Sinne Gegenstand der Rüge und der Klage sein kann, ist umstritten. In seiner Entschließung zum Zustimmungsgesetz zum Vertrag von Lissabon vom 23. April $2008^{53}$ hatte sich der Bundestag zwar unter Ver-

48 Gemäß §61 GO-BT legt der Ausschussvorsitzende die Tagesordnung fest, die der Ausschuss mit Mehrheit ändern kann.

49 Interparliamentary EU Information Exchange. Siehe die Internetseite von IEPX unter: www.ipex.eu (letzter Zugriff: 22.09.2010).

50 Die an IPEX weitergegebenen Informationen über den Stand der Beratungen sind auf der Internetseite von IPEX öffentlich abrufbar.

51 Eine wichtige Rolle kommt dabei den Fraktionen zu, die auf Arbeitsebene Initiativen vorbereiten, zwischen den Fachpolitikern vermitteln und Kontakte zu anderen Parlamenten herstellen könnten. Ein mögliches Modell dafür bietet die Ansiedlung eines eigens mit der Subsidiaritätsprüfung befassten Mitarbeiters beim Ersten Parlamentarischen Geschäftsführer der CDU-Fraktion seit Mai 2009. Die übrigen Fraktionen haben eine solche Koordinierungsstelle bislang nicht eingerichtet.

52 Artikel 5 Absatz 3 EUV.

53 Vgl. Bericht und Beschlussempfehlung des Europaausschusses, 2008, Ziffer 2 der Beschlussempfehlung. 
weis auf die Leitlinien gemäß Ziffer 5 des Protokolls über die Anwendung der Grundsätze der Subsidiarität und Verhältnismäßigkeit zum Vertrag von Amsterdam für eine umfassende Subsidiaritätsprüfung unter Berücksichtigung der Kompetenzgrundlage und der Verhältnismäßigkeit ausgesprochen. In jüngster Zeit waren an dieser Interpretation jedoch erneut Zweifel aufgetreten. ${ }^{54}$

Um für die Anwendung der Subsidiaritätsrüge und -klage eine bessere Grundlage und ein fraktionsübergreifendes Verständnis zu fördern, veranstaltete der Unterausschuss Europarecht des Rechtsausschusses im Deutschen Bundestag am 16. Juni 2010 ein Expertengespräch über die „Prüfung des unionsrechtlichen Subsidiaritätsprinzips“, das aber lediglich die Bandbreite der unterschiedlichen juristisch vertretenen Auffassungen offenbarte. Der Konsens hinsichtlich der Definition des Subsidiaritätsprinzips fand sich lediglich darin, dass eine belastbare Definition nach Auffassung der meisten Sachverständigen erst aus künftigen Urteilen des Europäischen Gerichtshofs (EuGH) über Subsidiaritätsklagen zu erwarten sei. Zugleich warnte der Sachverständige Wolfgang Spoerr in seiner Stellungnahme zum Expertengespräch des Unterausschusses Europarecht vor zu hohen Erwartungen an die definierende Rolle des EuGH in dieser Frage. Es erscheine vielmehr durchaus plausibel, dass der EuGH die Auslegung des Subsidiaritätsprinzips ähnlich wie die Rechtsprechung des amerikanischen Supreme Court zur Interstate Commerce Clause oder des Bundesverfassungsgerichts zur Erforderlichkeitsklausel in Artikel 72 Absatz 2 GG für eine effektive Ausgestaltung des institutionellen und föderalen Gleichgewichts nutzen könnte. Dabei ist aber auch zu beachten, dass gerade die Rechtsprechung des Supreme Court im Laufe der Zeit dieses Gleichgewicht immer wieder unterschiedlich interpretiert hat. ${ }^{55}$

\section{Subsidiaritätsklage}

Die Subsidiaritätsklage ${ }^{56}$ ist im Gegensatz zu der Subsidiaritätsrüge ein deutlich schärferes Schwert, da eine erfolgreiche Klage die Neufassung des betroffenen Gesetzgebungsaktes erforderlich machen würde. Durch die Ausgestaltung als Minderheitenrecht in dem neuen Absatz 1a des Artikels 23 GG und in $\S 12$ des IntVG könnte schon eine der großen Fraktionen alleine eine Klage veranlassen, ohne sich zuvor in irgendeiner Weise Unterstützung aus anderen nationalen Parlamenten sichern zu müssen. Im Gegensatz zur Subsidiaritätsrüge ist das Verfahren zur Erhebung einer Subsidiaritätsklage sehr einfach, weshalb davon auszugehen ist, dass eine solche Klage eher früher als später irgendwo in der Europäischen Union erhoben wird.

Unbefriedigend bleibt dabei, dass auch die Klage keine wirklichen Gestaltungsmöglichkeiten jenseits der reinen Verhinderung bietet. Nun kann in der Regel davon ausgegangen werden, dass entgegen der Mär von unnötiger Überregulierung die meisten Initiativen auch auf Ebene der Europäischen Union einem gewissen, zumindest subjektiv empfundenen oder sogar objektiv festgestellten Handlungsbedarf folgen. Es ist deshalb auch selten der Fall, dass nach der Aufhebung eines Gesetzes durch beispielsweise das Bundesverfassungsgericht keinerlei ,Ersatz' geschaffen wird. Vielmehr zieht eine verfassungsrechtliche Verwerfung in aller Regel eine Neufassung des betreffenden Gesetzgebungsaktes nach sich. Gleiches wird auch nach einer erfolgreichen Subsidiaritätsklage passieren. Im besten Falle

54 Für eine Übersicht dieser Diskussion siehe u.a. Franz C. Mayer: Stellungnahme zum öffentlichen Expertengespräch „Prüfung des unionsrechtlichen Subsidiaritätsprinzips“ im Unterausschuss Europarecht des Rechtsausschusses des Deutschen Bundestages am 16. Juni 2010, 09.06.2010, S. 16-18.

55 Wolfgang Spoerr: Thesen zur Expertenanhörung des Unterausschusses Europarecht „Prüfung des unionsrechtlichen Subsidiaritätsprinzips“ im Unterausschuss Europarecht des Rechtsausschusses des Deutschen Bundestages am 16. Juni 2010, 15.09.2010, S. 1-2.

56 Artikel 8 Subsidiaritätsprotokoll. 
könnte der EuGH mit dem Urteil Wege aufweisen, wie die verfolgte Zielsetzung unter Berücksichtigung der Subsidiarität besser erreicht werden kann. Im ungünstigsten Falle könnte ein Urteil dazu führen, dass gemeinsamem europäischen Handlungsbedarf keine rechtliche Antwort entgegengesetzt werden kann. Politisch wäre dies so oder so für die gegebenen Mehrheiten unbefriedigend. Für die nationalen Parlamente ist die Subsidiaritätsklage eine Möglichkeit, ihre Anliegen zu Gehör zu bringen. Eine Garantie, dass sie letztlich Beachtung finden, stellt die Klagemöglichkeit jedoch nicht dar. ${ }^{57}$ So offenbart sich auch die Subsidiaritätsklage politisch nur als Hilfsmittel in der politischen Auseinandersetzung.

\section{Die Anwendung des Gesetzes über die Zusammenarbeit von Bundesregierung und Bundestag in Angelegenheiten der Europäischen Union (EUZBBG)}

\section{Stärkung der Informations- und Mitwirkungsrechte im europapolitischen Alltagsgeschäft}

Im Gegensatz zu dem IntVG wurde für das EUZBBG schon 1992 mit der Neufassung des Artikels 23 GG die Grundlage geschaffen. Das Gesetz selbst wurde im März 1993 ausgefertigt. Mit dem Begleitgesetz zum Vertrag von Lissabon wurde dieses Gesetz novelliert. Die Neuerungen im Gesetz waren weitestgehend schon Bestandteil der EUZBBV gewesen oder bauten auf den Erfahrungen auf, die im Rahmen der Umsetzung der EUZBBV bereits gesammelt worden waren. ${ }^{58}$ Durch die gesetzliche Regelung bekamen die Bestimmungen der EUZBBV jedoch eine neue rechtliche Form und zusätzliche politische Bedeutung.

Die Europatauglichkeit des Bundestages war seit dem Inkrafttreten der EUZBBV Ende 2006 schon deutlich verbessert worden. Die EUZBBV war ursprünglich Teil des Gesetzespaketes zur Ratifizierung des Vertrages über eine Verfassung für Europa gewesen. Während der größte Teil dieses Paketes durch das Scheitern der Referenden in den Niederlanden und Frankreich hinfällig wurde, blieb die Abmachung zur Schaffung einer Zusammenarbeitsvereinbarung gültig. In der Abmachung waren auch schon wesentliche Inhalte enthalten, die in einer solchen Vereinbarung enthalten sein sollten. ${ }^{59}$ Hierzu gehörten insbesondere der verbesserte Zugang zu vorbereitenden Dokumenten der EU-Institutionen und der Bundesregierung sowie die Ausgestaltung der Mitwirkungsverfahren.

Alleine der erleichterte Zugang zu Informationen, insbesondere zu den Ratsdokumenten und zu den Drahtberichten, in denen die Vertreter der Bundesregierung über die vorbereitenden Gremien des Rates an die Ministerien in Berlin Bericht erstatten, hat ein sehr viel aktiveres und eigenständigeres europapolitisches Engagement der Abgeordneten ermöglicht. Das befürchtete beziehungsweise vorhergesagte ,Ertrinken ' in der Informationsflut ist ausgeblieben. Die rund 30 Mitarbeiterinnen und Mitarbeiter des Referates PA1 der Bundestagsverwaltung ${ }^{60}$ sowie ihre Kolleginnen und Kollegen im Verbindungsbüro des Deutschen Bun-

$57 \mathrm{Zu}$ der zu erwartenden Vorwirkung der Subsidiaritätskontrolle durch die nationalen Parlamente siehe Mayer: Stellungnahme, 2010, S. 19-20.

$58 \mathrm{Zu}$ den Neuerungen, die sich aus der EUZBBV ergaben, und den damit verbundenen Erwartungen siehe Axel Schäfer/Michael Roth/Christoph Thum: Stärkung der Europatauglichkeit des Bundestages, in: integration 1/2007, S. 44-49. Die Mängel bei der Umsetzung der EUBBV sind in den folgenden Anträgen dokumentiert. Vgl. Antrag der Abgeordneten Rainder Steenblock u.a. und der Fraktion Bündnis 90/Die Grünen. Zwei Jahre Europa-Vereinbarung, 2009; Antrag der Fraktionen der CDU/CSU, SPD und FDP. Vereinbarung über Zusammenarbeit in Angelegenheiten der Europäischen Union, 2009.

59 Vgl. Schäfer/Roth/Thum: Stärkung der Europatauglichkeit, 2007, S. 46-49.

60 Das Referat PA1 der Bundestagsverwaltung ist für die Beobachtung und Aufbereitung von EU-Vorlagen, die Verbindung zur Bundesregierung in Angelegenheiten der Europäischen Union, die Auskunftserteilung zu grundsätzlichen Fragen der Mitwirkung in EU-Angelegenheiten und die interparlamentarische Zusammenarbeit in EU-Angelegenheiten zuständig. 
destages in Brüssel haben durch die Priorisierung von Dokumenten, durch die Berichte und Kurzinformationen über aktuelle Entwicklungen wesentlich dazu beigetragen, die Informationsfülle politisch handhabbar zu machen. Die Fraktionen haben ihrerseits Personal nach Brüssel entsandt. Technisch wurde mit einer Ordnerstruktur im Intranet des Deutschen Bundestages eine praktikable Übergangslösung geschaffen, die voraussichtlich bis Ende 2010 durch ein umfassendes und vielversprechendes System des Dokumentenmanagements mit der Bezeichnung „Eudox“ ersetzt werden soll. Dieses soll die dem Bundestag von der Bundesregierung zugesandten Dokumente überschaubarer und nach Dossiers sowie Ereignissen zugänglich machen. ${ }^{61}$

Lückenlos und fehlerfrei ist die Umsetzung der Informationspflichten auch mit dem neuen EUZBBG noch nicht. So bleiben Probleme etwa bei der Berichterstattung über Ratsarbeitsgruppen im sogenannten ,Hauptstadtformat', an denen nicht Beamte der Ständigen Vertretung in Brüssel teilnehmen sondern die zuständigen Referentinnen oder Referenten des jeweiligen Ressorts aus Berlin oder Bonn. Hierüber werden nach Angaben der Bundesregierung keine Berichte verfasst, sodass dem Bundestag auch nichts zugesandt werden kann. Auch die Übermittlung von Beratungsdokumenten erfolgt aus Sicht der Abnehmer im Bundestag nicht immer in der wünschenswerten Geschwindigkeit. Insgesamt kann aber die enorme Verbesserung des Informationsstandes nicht bezweifelt werden. Im Großen und Ganzen erhält der Bundestag heute ausreichende Informationen, um die Verhandlungsprozesse im Rat und im Europäischen Parlament nachvollziehen zu können und Lücken in der Berichterstattung zu erkennen. Damit ist dann auch eine gute Grundlage für mündliche oder schriftliche Nachfragen gegeben.

Im Ergebnis steht diesem enormen Input an Informationen und Dokumenten allerdings eine relativ geringe Zahl an Stellungnahmen des Bundestages gegenüber, auch wenn ihre Zahl seit dem Inkrafttreten des Vertrages von Lissabon zweifellos bemerkbar zugenommen hat. Ursache für diese Zurückhaltung ist einerseits die Mittelbarkeit der politischen Einflussmöglichkeiten des Bundestages auf europäische Entscheidungen und andererseits das enge Verhältnis zwischen der Parlamentsmehrheit und der Bundesregierung im politischen System der Bundesrepublik Deutschland.

Die Mitwirkung des Bundestages im Sinne einer Mitgestaltung europapolitischer Entscheidungen findet auch nach dem Inkrafttreten des Vertrages von Lissabon vor allem über die Bundesregierung statt, die in Brüssel am Verhandlungstisch sitzt. ${ }^{62}$ Auf sie wirken der Bundestag und seine Mitglieder in Form von Stellungnahmen und durch die Beteiligung an Diskussionsprozessen über Partei und Öffentlichkeit ein. Im Gegensatz zu dem ansonsten für Abgeordnete des Deutschen Bundestages gewohnten direkten Einfluss auf Rechtsetzung und Entscheidungsfindung im Parlament handelt es sich bei der Europapolitik somit stets um einen mittelbaren Einfluss. Dadurch wird das politische Engagement sehr viel weniger attraktiv. Dem gleichen oder gar größeren Aufwand steht eine geringere Wirkung gegenüber.

Hinzu kommt, dass in parlamentarischen Regierungssystemen Regierung und Parlamentsmehrheit politisch eng miteinander verbunden sind. Daher kommt den informellen Kommunikationsprozessen innerhalb der Regierungsfraktionen und zwischen den Regierungsfraktionen und der Regierung eine besondere Bedeutung zu, die zu formalen Beschlüs-

61 Eine anschauliche Darstellung der Art und Weise, wie der Informationsfluss von EU-Dokumenten im Bundestag abläuft bietet der Artikel von Annette Sach: Die Europa-Sensoren, in: Das Parlament, 03.05.2010.

62 Diese Haltung wird auch in der Entschließung zum Zustimmungsgesetz zum Vertrag von Lissabon vom 23. April 2009 zum Ausdruck gebracht. Vgl. Ziffer 2 der Beschlussempfehlung und Bericht des Ausschusses für die Angelegenheiten der Europäischen Union, 2008, S. 4. 
sen eine effizientere Alternative bieten. Die Zahl der formalen Stellungnahmen des Bundestages zu EU-Vorhaben ist insofern kein verlässliches Maß für den Einfluss der Parlamentarierinnen und Parlamentarier auf die deutsche Verhandlungsposition im Rat. Hierfür ist ebenso wichtig, wie häufig die zuständigen Ministerinnen und Minister beziehungsweise Staatssekretäre oder gar zuständige Ministerialbeamte an den Sitzungen der Arbeitsgruppen und Arbeitskreise im Bundestag teilnehmen und die dort zum Ausdruck gebrachten Positionen bei den Verhandlungen im Rat berücksichtigen.

Die Zahl der Stellungnahmen ist lediglich für das Selbstverständnis des Bundestages mit Blick auf europäische Politik von Bedeutung. Sie können nach Außen kommuniziert und in öffentlichen Debatten als glaubhafter Beweis für das politische Engagement in der jeweiligen Sache angeführt werden. Ohne Zweifel wächst in den Fraktionen das Bewusstsein, dass die europäische Ebene für den Bundestag relevant ist und dass sie der Bundesregierung bei den Verhandlungen im Rat auf die Finger schauen müssen, wenn sie nicht von unerwünschten Ergebnissen überrascht werden möchten. Dies geschieht im kleinen Kreise aber gelegentlich sogar leichter, als in der Öffentlichkeit des Plenarsaals.

Anders verhält es sich mit den Oppositionsfraktionen. Sie sind die eigentlichen Gewinner der europapolitischen Stärkung des Bundestages, insbesondere mit Blick auf die Informationsrechte, die ihnen zunächst die EUZBBV und nun das neue EUZBBG sichern. ${ }^{63}$ Gerade bei der Opposition hat dementsprechend die Anzahl der Anträge zu EU-Themen deutlich zugenommen. Den parlamentarischen Mehrheitsverhältnissen entsprechend werden diese Anträge in der Regel jedoch als Oppositionsanträge abgelehnt oder in seltenen Fällen zugunsten von fraktionsübergreifenden Anträgen zurückgezogen.

Die Formulierung von Stellungnahmen zu EU-Vorhaben ist aber kein Selbstzweck. Dies gilt in besonderem Maße für die Opposition, die in Ermangelung der informellen Einflusskanäle die Bundesregierung beziehungsweise die Koalitionsfraktionen mit ihren Anträgen zur öffentlichen Positionierung drängen kann.

Grundsätzlich kann der Bundestag mit seinen Stellungnahmen die Bundesregierung in ihrer Verhandlungsführung beziehungsweise in ihrem Abstimmungsverhalten im Rat im jeweils eigenen Sinne beeinflussen. Binden kann der Bundestag die Bundesregierung an seine Position allerdings nicht. Artikel 23 Absatz 3 Satz 2 GG lautet: „Die Bundesregierung berücksichtigt die Stellungnahme des Bundestages bei den Verhandlungen." ${ }^{64}$ Bestrebungen, eine festere Bindung der Bundesregierung im Sinne eines imperativen Mandats, also einer Art Weisungsrecht, einzuführen, wurden im Gesetzgebungsverfahren vor allem von der CSU immer wieder gefordert. Nicht nur die Notwendigkeit einer Grundgesetzänderung sprach dagegen. Vielmehr bescheinigt das Bundesverfassungsgericht ja auch explizit der Bundesregierung eine Integrationsverantwortung, die sie allerdings nur innerhalb eines gewissen Ermessensraumes wahrnehmen kann. ${ }^{65}$

Es geht in der Ausgestaltung der Mitwirkungsrechte des Bundestages also um eine Balance zwischen der Eigenverantwortung der Bundesregierung für ihr Handeln im Rat und den Mitwirkungsmöglichkeiten des Bundestages beziehungsweise des Bundesrates. Schon in der EUZBBV wurde diese Balance durch ein detailliertes Verfahren geregelt, demzufolge die Bundesregierung die Stellungnahmen des Bundestages ihren Verhandlungen zugrunde-

63 Vgl. Wolfgang Ismayr: Der Deutsche Bundestag seit 1990, in: Aus Politik und Zeitgeschichte 28/2009, S. 3440.

64 Hervorhebung des Autors.

65 Vgl. Redebeitrag von Christian Calliess in der 90. Ausschusssitzung des Ausschusses für die Angelegenheiten der Europäischen Union. Vgl. Deutscher Bundestag: Protokoll der 90. Sitzung des Europaausschusses, 2009, S. 47-48. 
zulegen und einen Parlamentsvorbehalt einzulegen hat, wenn die in der Stellungnahme aufgeführten wesentlichen Belange des Bundestages nicht durchgesetzt werden können. Unter diesem Vorbehalt kann die Bundesregierung dem Verhandlungsergebnis zustimmen, sie muss sich vor der abschließenden Entscheidung im Rat aber um die Herstellung des Einvernehmens mit dem Bundestag bemühen. ${ }^{66}$ Dieses Verfahren war in Ziffer II der EUZBBV geregelt, die weitgehend unverändert in $\S 9$ EUZBBG übernommen wurde. Auch für die Aufnahme von Verhandlungen zur Vorbereitung von Beitritten oder Vertragsänderungen muss die Bundesregierung gemäß $§ 10$ EUZBBG das Einvernehmen mit dem Bundestag herstellen.

Auslegungsschwierigkeiten bei der Herstellung des Einvernehmens gemäß $\S 10$ Absatz 2 Satz 2 EUZBBG

Unklar ist bei der Anwendung von § 10 EUZBBG jedoch, ob das Einvernehmen bei jeder Entscheidung über die Eröffnung von Verhandlungen über Beitritte oder Vertragsänderungen hergestellt werden muss oder ob dies nur dann gilt, wenn der Bundestag zuvor eine entsprechende Stellungnahme abgegeben hat. Diese Unklarheit wurde gleich im ersten Halbjahr nach Inkrafttreten des Vertrages von Lissabon deutlich. Schon am 4. Dezember 2009, drei Tage nach dem Inkrafttreten des Vertrages von Lissabon, legte die spanische Ratspräsidentschaft den ersten Vorschlag zur Aufnahme von Verhandlungen über die vertraglichen Grundlagen der Europäischen Union vor ${ }^{67}$ und am 24. Februar 2010 legte die Europäische Kommission ihren ,Avis` zum Beitrittsgesuch Islands vom 22. Juli 2009 vor, der als Vorlage für die Eröffnung der Beitrittsverhandlungen dienen sollte.

$\S 10$ des EUZBBG geht von der Unterrichtung der Bundesregierung über Vorschläge und Initiativen für Beschlüsse zur Aufnahme von Verhandlungen zur Vorbereitung eines Beitrittes zur Europäischen Union aus, in deren Zusammenhang die Bundesregierung den Bundestag auf sein Recht zur Stellungnahme ,nach § 9“ hinzuweisen hat. Vor der abschließenden Entscheidung im Rat, gemäß Absatz 2, solle die Bundesregierung Einvernehmen mit dem Bundestag herstellen. Das Recht der Bundesregierung, in Kenntnis der Stellungnahme des Bundestages aus wichtigen außen- und integrationspolitischen Gründen abweichende Entscheidungen zu treffen, bleibe unberührt.

66 Vgl. hierzu Schäfer/Roth/Thum: Stärkung der Europatauglichkeit, 2007, S. 48.

67 Hintergrund war die Vereinbarung der Staats- und Regierungschefs vom 11. und 12. Dezember 2008, Übergangsmaßnahmen zur vollen Ausschöpfung der im Vertrag von Lissabon vereinbarten Mandatszahlen aller Mitgliedstaaten zu ergreifen, sollte der Vertrag erst nach den Wahlen zum Europäischen Parlament im Juni 2009 in Kraft treten. Diese Vereinbarung wurde auf dem Europäischen Rat vom 18. und 19. Juni 2009 konkretisiert. Da die Wahlen zum Europäischen Parlament vom 4. bis 7. Juni 2009 noch gemäß den Regelungen des Vertrages von Nizza stattfanden, konnte die im Vertrag von Lissabon vorgesehene Änderung der Sitzzahl und -verteilung bei der Vergabe der Mandate nicht mehr berücksichtigt werden. Um den betroffenen Mitgliedstaaten die Zustimmung zu den nach dem irischen Referendum vorgenommenen Ergänzungen zu ermöglichen, hatten sich die Staats- und Regierungschefs auf eine Erhöhung der Sitze des Europäischen Parlaments von 736 um 18 Abgeordnete aus zwölf EU-Mitgliedstaaten auf 754 Sitze bis zum Ende der laufenden Legislaturperiode im Jahr 2014 geeinigt. Auf Grundlage dieses rechtlich unverbindlichen Beschlusses unterbreitete die spanische Regierung dem Rat der Europäischen Union am 4. Dezember 2009 einen Vorschlag für Übergangsmaßnahmen betreffend die Zusammensetzung des Europäischen Parlaments. Durch eine Änderung des Protokolls Nr. 36 zum Vertrag von Lissabon sollen diejenigen EU-Mitgliedstaaten zusätzliche Sitze im Europäischen Parlament erhalten, die eine größere Zahl an Abgeordneten gehabt hätten, wäre der Vertrag von Lissabon zum Zeitpunkt der Wahlen zum Europäischen Parlament bereits in Kraft gewesen. Vgl. Rat der Europäischen Union: Änderung der Verträge - Übergangsmaßnahmen betreffend die Zusammensetzung des Europäischen Parlaments, Dok. 17196/09. 
Für die Auslegung dieser Regelung gibt es zwei mögliche Lesarten:

1. Der Verweis auf $\S 9$ EUZBBG stellt klar, dass für den Sachverhalt des $\S 10$ EUZBBG die in $\S 9$ festgelegten Verfahren gelten. Demnach muss das Einvernehmen nur hergestellt werden, wenn die Bundesregierung einem Vorschlag zustimmen möchte, obwohl nicht alle in einer Stellungnahme beschlossenen wesentlichen Belange des Bundestages durchgesetzt werden konnten.

2. Der Verweis auf $§ 9$ EUZBBG dient lediglich der Klarstellung, dass der Bundestag zu Vorschlägen zur Aufnahme von Verhandlungen über Beitritte oder Vertragsänderungen ebenso das Recht zur Stellungnahme hat, wie zu sonstigen Vorhaben. Der zweite Absatz von § 10 EUZBBG ist hiervon jedoch nicht betroffen, sodass die Bundesregierung unabhängig von einer solchen Stellungnahme vor der abschließenden Entscheidung im Rat das Einvernehmen mit dem Bundestag herstellen soll.

Während SPD und Bündnis 90/Die Grünen bei der Diskussion über den spanischen Vorschlag zur Vertragsveränderung und den Umgang mit dem Beitrittsgesuch Islands im Europaausschuss die erste der beiden Positionen vertraten, legten sich die Vertreter der Koalition auf die Zweite fest. SPD und Bündnis 90/Die Grünen brachten auf der Grundlage ihres Verständnisses schon im Dezember 2009 Anträge zu dem isländischen Beitrittsgesuch ein, ${ }^{68}$ in denen die Bundesregierung aufgefordert wurde, aktiv das Einvernehmen mit dem Bundestag zu suchen und herzustellen (Antrag der Fraktion Bündnis 90/Die Grünen) beziehungsweise den Bundestag um die Herstellung des Einvernehmens zu bitten (Antrag der SPD-Fraktion). Die Fraktion Bündnis 90/Die Grünen forderte zusätzlich eine Regierungserklärung vor der abschließenden Entscheidung im Rat. Ein Antrag der Koalitionsfraktionen von CDU, CSU und FDP wurde am 24. März 2010 eingebracht, mit dem das nach Auffassung der Koalitionsfraktionen unabhängig von einer vorausgegangenen Stellungnahme erforderliche Einvernehmen hergestellt wurde. ${ }^{69}$

Im Rahmen der Debatte im Europaausschuss am 24. März 2010 wurde die Bedeutung der unterschiedlichen Auffassungen deutlich: In Ermangelung einer Stellungnahme zum Avis stellte die Fraktion der SPD im Ausschuss den Antrag, dass das Ausstehen eines Einvernehmens zwischen Bundestag und Bundesregierung bezüglich der Eröffnung von Beitrittsverhandlungen festgestellt werden sollte. Begründet war dieses auf den ersten Blick kleinliche Vorgehen mit der Sorge, die Bundesregierung könne diesen ersten Anwendungsfall des $§ 10$ EUZBBG zum Präzendenzfall machen und somit die neu erworbenen Rechte des Bundestages durch eine schnelle Zustimmung ohne vorige Stellungnahme und ohne Herstellung des Einvernehmens wieder beschneiden. ${ }^{70}$ Hintergrund war, dass noch in der 16 . Wahlperiode die Bundesregierung die Auffassung vertreten hatte, nach der Unterrichtung der Bundesre-

68 Antrag der Fraktion der SPD. Herstellung des Einvernehmens über die Aufnahme von Verhandlungen über den Beitritt der Republik Island zur Europäischen Union, Bundestagsdrucksache 17/246 vom 15. Dezember 2009; Antrag der Abgeordneten Manuel Sarrazin u.a. und der Fraktion Bündnis 90/Die Grünen. Rechte des Bundestages nach den Begleitgesetzen zum Vertrag von Lissabon wahren. hier: Einvernehmen mit dem Bundestag vor der Aufnahme von Beitrittsverhandlungen mit Island herstellen, Bundestagsdrucksache 17/260 vom 16. Dezember 2009.

69 Antrag der Fraktionen der CDU/CSU und FDP. Einvernehmensherstellung von Bundestag und Bundesregierung zum Beitrittsantrag der Republik Island zur Europäischen Union und zur Empfehlung der EU-Kommission vom 24. Februar 2010 zur Aufnahme von Beitrittsverhandlungen. hier: Stellungnahme des Deutschen Bundestages nach Artikel 23 Absatz 3 GG i. V. m. § 10 des Gesetzes über die Zusammenarbeit von Bundesregierung und Deutschem Bundestag in Angelegenheiten der Europäischen Union, Bundestagsdrucksache 17/ 1190 vom 24. März 2010.

70 Vgl. Beschlussempfehlung und Bericht des Ausschusses für die Angelegenheiten der Europäischen Union (21. Ausschuss) [...] b) zu dem Entschließungsantrag der Abgeordneten Dietmar Nietan, u.a und der Fraktion der SPD - Drucksache 17/1191- [...], Bundestagsdrucksache 17/1464 vom 21. April 2010. 
gierung über die Aufnahme von Verhandlungen über Beitritte zur Europäischen Union oder zur Änderung der vertraglichen Grundlagen der Europäischen Union obliege es dem Deutschen Bundestag zu entscheiden, zu dieser Unterrichtung nach Ziffer VI der EUZBBV Stellung zu nehmen..$^{71}$ Ohne eine weitere Stellungnahme hätte das Einvernehmen bestanden und die Bundesregierung hätte dem Vorschlag im Rat zustimmen können. In dem Fall des isländischen Beitritts war die Zustimmung an sich unstrittig. Im Ergebnis wurde der entsprechende Antrag der Koalitionsfraktionen noch vor der abschließenden Entscheidung im Rat angenommen, sodass der Präzedenzfall nun dafür spricht, dass auch in Zukunft immer zunächst das Einvernehmen herzustellen ist.

Eine letzte Unsicherheit bleibt mit Blick auf die Frage, auf welche ,abschließende Entscheidung im Rat" sich $§ 10$ Absatz 2 EUZBBG bezieht. Geht es hierbei um die abschlieBende Entscheidung über die Aufnahme von Verhandlungen oder über die Annahme der Verhandlungsergebnisse? Letzteres würde bedeuten, dass nach Abschluss von Verhandlungen über Vertragsänderungen noch einmal das Einvernehmen mit dem Bundestag hergestellt werden müsste. Diese Auffassung fand in dem Antrag der Koalitionsfraktionen zu dem Vorschlag der spanischen Regierung für Übergangsmaßnahmen betreffend die Zusammensetzung des Europäischen Parlaments ${ }^{72}$ Niederschlag. Dabei wurde außer Acht gelassen, dass Artikel 48 Absatz 4 EUV nach der Einigung der Regierungskonferenz keine weitere Ratsbefassung vorsieht sondern nur noch die Ratifizierung durch die Mitgliedstaaten.

\section{Anpassung der Geschäftsordnung des Bundestages - verpasste Chance zur Verbesse- rung der Europatauglichkeit?}

Mit dem Inkrafttreten des Vertrages von Lissabon und der Begleitgesetze musste auch die Geschäftsordnung des Deutschen Bundestages teilweise redaktionell und teilweise mit Blick auf die neuen Verfahren und Möglichkeiten aus dem Vertrag und den Begleitgesetzen angepasst werden. ${ }^{73}$

Der Bundestag verfolgt im Gegensatz zu anderen nationalen Parlamenten in der Europäischen Union nicht das Modell eines Europa-Hauptausschusses, sondern vielmehr eine Art ,Europa-Mainstreaming ', das hier anhand der Zuständigkeit der Fachausschüsse für die Subsidiaritätsprüfung bereits dargestellt wurde. Die Fachausschüsse werden dadurch explizit zur Wahrnehmung ihrer europapolitischen Verantwortung im Rahmen ihrer regelmäßigen Ausschussarbeit verpflichtet. So wird vermieden, dass die Befassung mit EU-Vorlagen in das europapolitische Sondergremium Europaausschuss ausgelagert wird. Diese grundsätzliche Ausrichtung der Befassung mit EU-Vorhaben prägt auch die Eingliederung europapolitischer Themen in die Geschäftsordnung jenseits der Ausschussarbeit. Besonders deutlich wird dies

71 Brief des Staatsministers im Auswärtigen Amt Günter Gloser und des Parlamentarischen Staatssekretärs im Bundesministerium für Wirtschaft und Technologie Peter Hintze vom 26. Mai 2006 an die Obleute der Bundestagsfraktionen im Ausschuss für die Angelegenheiten der Europäischen Union.

72 Antrag der Fraktionen der CDU/CSU und FDP. Übergangsmaßnahmen zur Zusammensetzung des Europäischen Parlamentes nach Inkrafttreten des Vertrages von Lissabon. hier: Stellungnahme des Deutschen Bundestages nach Artikel 23 Absatz 3 GG i. V. m. § 10 des Gesetzes über die Zusammenarbeit von Bundesregierung und Deutschem Bundestag in Angelegenheiten der Europäischen Union, Bundestagsdrucksache 17/1179 vom 24. März 2010.

73 Die Änderung der Geschäftsordnung wurde mit der Annahme der Beschlussempfehlung und des Berichts des Ausschusses für Wahlprüfung, Immunität und Geschäftsordnung am 8. Juli 2010 angenommen. Alle Angaben zu den vorgenommenen Änderungen sind hieraus entnommen. Vgl. Beschlussempfehlung und Bericht des Ausschusses für Wahlprüfung, Immunität und Geschäftsordnung (1. Ausschuss). Änderung der Geschäftsordnung des Deutschen Bundestages. hier: Änderungen im Hinblick auf den Vertrag von Lissabon, Bundestagsdrucksache 17/2394 vom 5. Juli 2010. 
bei der äußerst restriktiven Ausgestaltung der Rechte des Europaausschusses zur Wahrnehmung seiner Kompetenz zur plenarersetzenden Beschlussfassung. Diese besteht zwar im Prinzip, da für einen plenarersetzenden Beschluss des Europaausschusses aber zunächst die Zustimmung des jeweils federführenden sowie Voten aller mitberatenden Ausschüsse eingeholt werden müssen, handelt es sich dabei um eine eher theoretische Möglichkeit. ${ }^{74}$

Diese starke Rolle der Fachausschüsse bei der Wahrnehmung der europapolitischen Mitwirkungsrechte des Bundestages mag nicht immer effizient erscheinen. Die Ausschüsse nutzen ihre Möglichkeiten in dieser Hinsicht sehr unterschiedlich und offenbaren dabei auch sehr unterschiedliche Wahrnehmungen der europäischen Integration, die bei den jeweiligen Ausschussmitgliedern vorherrschen. Die Erfahrung aus anderen Parlamenten zeigt jedoch, dass ein Europaausschuss als ,Hauptausschuss“ für alle europapolitischen Entscheidungen letztlich auch eine intensive Zuarbeit der Fachausschüsse braucht und somit nicht wirklich eigenständig mitzuwirken vermag. ${ }^{75}$ Kritisch ist vor dem Hintergrund der Achtwochenfrist bei der Subsidiaritätsrüge aber, inwiefern die plenarersetzenden Beschlusskompetenzen des Europaausschusses zumindest in dieser Beziehung hätten erleichtert werden können. Entsprechende Überlegungen fruchteten im zuständigen Ausschuss für Geschäftsordnung, Immunität und Wahlprüfung jedoch nicht.

Die wichtigsten Änderungen, auf die sich die Fraktionen in der jüngsten Anpassung der Geschäftsordnung in Hinblick auf das Inkrafttreten des Vertrages von Lissabon und seiner Begleitgesetze geeinigt haben, betreffen die Regelungen zur Erhebung einer Subsidiaritätsrüge ${ }^{76}$ sowie einer Subsidiaritätsklage ${ }^{77}$ durch den Deutschen Bundestag und die Regelung zur Delegation von Mitwirkungsrechten aus dem IntVG an den Europaausschuss. ${ }^{78}$ Erwähnt sei auch die Ergänzung eines Absatzes 4 in $§ 93 a$ GO-BT. Er stellt klar, dass für die Herstellung des Einvernehmens gemäß $§ 10$ Absatz 2 Satz 1 EUZBBG dasselbe Verfahren gilt, wie für das Einvernehmen bei Stellungnahmen gemäß § 9 Absatz 4 Satz 4 EUZBBG. Damit greift die Geschäftsordnung die von SPD und Bündnis 90/Die Grünen vertretene Ansicht hinsichtlich der Bedeutung von Stellungnahmen im Vorfeld von Verhandlungen über Beitritte und Vertragsänderungen auf (siehe oben) und verdeutlicht, dass das Einvernehmen nur durch das Plenum des Bundestages und nicht durch einen Ausschuss hergestellt werden kann. Das Recht auf eine Plenardebatte im Falle der Nichtdurchsetzung wesentlicher Belange gemäß § 9 Absatz 5 Satz 3 EUZBBG wurde mit der Einfügung eines neuen Absatzes 8 in § 93 GO-BT ebenso wie die Subsidiaritätsklage als Minderheitenrecht angelegt. Während für letzteres ein Viertel der Mitglieder des Bundestages erforderlich sind, reicht für ersteres das Verlangen einer Fraktion oder 5 Prozent der Abgeordneten.

Ein wichtiges Thema bei der Diskussion über die Änderung der Geschäftsordnung war die Fähigkeit zur kurzfristigen Reaktion, insbesondere in der sitzungsfreien Zeit. Klar ist, dass der Bundestag auch außerhalb der Sitzungswochen handlungsfähig sein muss, zumal sowohl die Subsidiaritätsprüfung als auch die Begleitung sonstiger Rechtsetzungsakte unter Berücksichtigung von Fristvorgaben der europäischen Ebene stattfinden. Insbesondere bei den Oppositionsfraktionen hatte es Befürworter dafür gegeben, konkrete Fristen in die Geschäftsordnung einzufügen, nach deren Ablauf die Federführung automatisch an den Europaausschuss überginge. Hintergrund war, dass die Koalition ihre Geschäftsordnungsmehr-

$74 \S 93$ b GO-BT.

75 Vgl. Carsten Schymik: Der Europaausschuss des dänischen Folketing - der machtvollste Europas?, FriedrichEbert-Stiftung: Internationale Politikanalyse, September 2008.

$76 \S 93$ c GO-BT.

$77 \S 93 \mathrm{~d}$ GO-BT.

$78 \S 93$ b GO-BT. 
heit in jedem Ausschuss nutzen kann, um koalitionsstrittige Abstimmungen zu vermeiden. Gerade bei Anträgen auf Subsidiaritätsrügen kann dies dazu führen, dass sich ein Antrag durch Ablauf der Achtwochenfrist erledigt. Entsprechende Vorstöße scheiterten jedoch an der bereits beschriebenen Abneigung gegen die Stärkung des Europaausschusses auf Kosten der Fachausschüsse. Umgekehrt stellt sich aber auch die Frage, ob der Europaausschuss in der Lage wäre, bei einer solchen Fristregelung unter erheblichem Zeitdruck komplexe Materien von den Fachausschüssen zu übernehmen und entsprechende Beschlüsse zu fassen.

Das Anliegen wurde in abgeschwächter Form in § 93 a Absatz 1 GO-BT aufgenommen, der nun den Hinweis enthält, die Ausschüsse sollten bei ihrer Beschlussfassung die auf der Ebene der Europäischen Union maßgeblichen Fristvorgaben berücksichtigen. Damit sind die acht Wochen für die Subsidiaritätsrüge nicht explizit genannt, indirekt aber berücksichtigt. Zugleich bietet die Formulierung eine Grundlage, auch in anderen Fällen eine schnelle Befassung zu erreichen, so etwa bei bevorstehender Beschlussfassung im Rat.

Die Anpassung der Geschäftsordnung hat die Europatauglichkeit des Bundestages somit nicht grundlegend revolutioniert, sie hat vielmehr notwendige Anpassungen unternommen, um die bestehenden Möglichkeiten für die Wahrnehmung von Mitwirkungsrechten an den Vertrag von Lissabon anzupassen. Ein Paradigmenwechsel hin zu einer dezidierten Stärkung des Europaausschusses hätte nicht nur Unmut bei den übrigen Ausschüssen ausgelöst, es ist auch alles andere als sicher, dass hierdurch die Europatauglichkeit des Bundestages als ganzes gestärkt worden wäre.

\section{Ausnahmen bei Eilverfahren?}

Besonders deutlich wurde das Spannungsverhältnis zwischen Handlungsfähigkeit der Bundesregierung einerseits und dem demokratischen Legitimationsanspruch des Bundestages bei der Verabschiedung des Euro-Stabilisierungspaketes vom 7. bis 9. Mai 2010. Am Abend des 7. Mai 2010, nachdem Bundeskanzlerin Angela Merkel am Vormittag noch in Berlin im Plenum des Bundestages an der Debatte über die Griechenlandhilfen teilgenommen hatte, wurde sie auf der Tagung des Europäischen Rats mit der Entscheidung über ein elfmal so großes Rettungspaket überrascht, das nach Auffassung der Kommission, der Europäischen Zentralbank und anderer Mitgliedstaaten aufgrund des , austrocknenden“ Interbankenhandels und der davon ausgehenden Gefahr für die Wirtschaft der Eurozone notwendig geworden sei. Zweifelsohne war es das Recht der Bundeskanzlerin und der Bundesregierung, wenn nicht gar ihre Pflicht, in dieser Situation in der nach ihrer Ansicht notwendigen Geschwindigkeit zu handeln. Für den Bundestag bedeutete dies jedoch, gleich zweimal in kürzester Frist über erhebliche finanzielle Engagements entscheiden zu müssen und trotz schwerwiegender Bedenken und möglicherweise weitreichender Konsequenzen auf eine parlamentarische Prüfung im angemessenen Zeitrahmen zu verzichten.

Eine Reihe von Fragen bleibt offen, was die verfassungs- und europarechtliche Zulässigkeit des Europäischen Finanzstabilisierungsmechanismus angeht, insbesondere hinsichtlich der Rechtsgrundlage für die Verordnung über den europäischen Finanzstabilisierungsmechanismus, ${ }^{79}$ aber auch hinsichtlich des innerstaatlichen Ratifizierungsbedarfs des Rahmenvertrages über den Europäischen Finanzstabilisierungsmechanismus (EFSF). ${ }^{80}$ Weitere Be-

79 Verordnung (EU) Nr. 407/2010 des Rates vom 11. Mai 2010 zur Einführung eines europäischen Finanzstabilisierungsmechanismus, in: Amtsblatt der EU, Nr. L 118 vom 12. Mai 2010, S. 1-4.

80 Zur Rechtsgrundlage der Verordnung 407/2010 vgl. Martin Seidel: Aktuelle Probleme der europäischen Währungsunion und ihre Bewältigung, in diesem Band der integration, S. 334-349; Thelmo Jeck: Euro-Rettungsschirm bricht EU-Recht und deutsches Verfassungsrecht, Centrum für Europäische Politik: CEP-Studie, 05.07.2010. 
denken bestehen hinsichtlich möglicher Auswirkungen der Verordnung auf den Haushalt der Europäischen Union, sollten die in der Verordnung enthaltenen Bürgschaften abgerufen werden. Kritisch ist auch, dass der Bundestag mit dem Verweis auf die Eilbedürftigkeit der Entscheidung entgegen der Vorgaben des Artikels 23 Absatz 3 GG im Falle der Verordnung 407/2010 keine Gelegenheit zur Stellungnahme vor der Mitwirkung der Bundesregierung an dem Rechtsetzungsakt bekam. Es bleibt abzuwarten, ob die von den Professoren Wilhelm Hankel, Joachim Starbatty, Karl Albrecht Schachtschneider und Wilhelm Nölling eingereichte Klage gegen den Euro-Rettungsschirm dem Verfassungsgericht als Anlass für eine weitere Stärkung des Bundestages dient. Zugleich ist die Frage nach den Konsequenzen gerechtfertigt, die insbesondere von Seiten der Fraktion Bündnis 90/Die Grünen gestellt wird. Als Reaktion auf die Vorgänge des Frühjahrs haben sie einen Gesetzentwurf zur nachträglichen Ratifizierung des EFSF-Vertrags ${ }^{81}$ sowie einen Antrag mit dem Titel „Unterrichtungsund Mitwirkungsrechte des Deutschen Bundestages in Bezug auf Europäische Räte stärken“ eingebracht. ${ }^{82}$ Ausgehend von der Feststellung, dass sich mit dem Inkrafttreten des Vertrages von Lissabon auch die Machtbalance innerhalb des Institutionengefüges der Europäischen Union ändert, werden hierin Konsequenzen für die Ausgestaltung der in EUZBBG und Geschäftsordnung festgelegten Mitwirkungsmöglichkeiten des Bundestages in Angelegenheiten der Europäischen Union gefordert. ${ }^{83}$ Im Kern geht es dabei um die bessere Durchsetzung der bestehenden Informationsrechte des Bundestages mit Blick auf die Tagungen des Europäischen Rates.

Dabei ist kritisch anzumerken, dass der Bundestag schon auf der Grundlage von Ziffer I.3 EUZBBV ein Anrecht auf umfassende Unterrichtungen zu jedem Beratungsgegenstand sowohl von Tagungen des Rates als auch des Europäischen Rates im Vorfeld der jeweiligen Sitzung hatte. Diese Informationsrechte wurden in § 5 Absatz 5 EUZBBG übernommen. Im konkreten Fall der Euro-Rettung im Mai 2010 bleibt unklar, ob die Bundesregierung tatsächlich nicht über die notwendigen Informationen verfügte oder ob sie diese dem Bundestag bewusst vorenthielt. Darüber hinaus ist aber auch klar, dass der Bundestag seine Informationsrechte im Zweifelsfalle stärker einfordern muss.

\section{Auf dem Weg zum Ziel}

Die Umsetzung der neuen Begleitgesetze zum Vertrag von Lissabon führt den Prozess der verbesserten Einbeziehung des Bundestages in die deutsche Europapolitik fort, die mit dem Inkrafttreten der Vereinbarung von Bundesregierung und Bundestag über die Zusammenarbeit in Angelegenheiten der Europäischen Union von 2006 eingeleitet wurde. Dieser Prozess zeigt viele Facetten, von der technischen Umsetzung des Dokumentenmanagements über die Anpassung der Geschäftsordnung bis hin zum Bewusstseinswandel und dem Anspruch der Abgeordneten, die in ihrem Arbeitsalltag zunehmend die europäische Ebene als Aktionsraum entdecken.

81 Gesetzentwurf der Abgeordneten Manuel Sarrazin u.a. und der Fraktion Bündnis 90/Die Grünen. Entwurf eines Gesetzes zu dem EFSF-Rahmenvertrag vom 7. Juni 2010, Bundestagsdrucksache 17/2412 vom 6. Juli 2010.

82 Antrag der Abgeordneten Manuel Sarrazin u.a. und der Fraktion Bündnis 90/Die Grünen. Unterrichtungs- und Mitwirkungsrechte des Deutschen Bundestages in Bezug auf Europäische Räte stärken, Bundestagsdrucksache $17 / 2437$ vom 7 . Juli 2010.

83 Zur sich verändernden Machtbalance innerhalb des europäischen Institutionengefüges vgl. auch Jean Paul Jacqué: Der Vertrag von Lissabon - neues Gleichgewicht oder institutionelles Sammelsurium?, in: integration 2/2010, S. 103-116. 
Das Inkrafttreten des Vertrages von Lissabon bringt eine Reihe neuer Entwicklungen mit sich - an erster Stelle die Stärkung des Europäischen Rates - die für den Bundestag eine zusätzliche Herausforderung darstellen. ${ }^{84}$ Auch die zunehmende Bedeutung der Europäischen Union als internationaler Akteur wird wesentliche Anpassungsprozesse in der Beratungsweise des Deutschen Bundestages erforderlich machen. Neben einem höheren Aufwand bei der Informationsgewinnung wird dabei auch die Berücksichtigung europäischer Zeitpläne und Beratungsabläufe an Bedeutung gewinnen. Der rechtliche Rahmen des Artikels 23 GG und des neuen EUZBBG bilden eine gute Ausgangsbasis und sollten offensiv genutzt werden. Nachdem über ein gutes Jahrzehnt auf europäischer Ebene die institutionelle Verfassungsdebatte die fachpolitischen Auseinandersetzungen in den Schatten gestellt hat, sollte sich die Europapolitik innerhalb Deutschlands nun keine Weiterführung dieser Nabelschau antun.

Die erheblichen Zweifel an der Rechtmäßigkeit des Vorgehens der Bundesregierung in der Euro-Krise zeigen, dass die politische Praxis schon auf der gegenwärtigen Basis mehr als genug Stoff bietet, um die Europatauglichkeit des Bundestages und dessen Mitwirkung weiter voran zu bringen. Dabei geht es in gleichem Maße um Anpassungen in den tatsächlichen Verfahren und Arbeitsmethoden wie auch um Veränderungen bei der Wahrnehmung und dem Verständnis politischer Prozesse. Eine zentrale Rolle wird dabei in Zukunft den Parteien und ihren Fraktionen zukommen, die sowohl bei der Organisation von politischen Abläufen als auch bei der Meinungsbildung maßgebliche Akteure sind. Die enge Verflechtung rechtlicher, wirtschaftlicher und somit auch politischer Zusammenhänge macht das Zusammenwirken der verschiedenen Ebenen heute immer dringender. Zwischen Bund und Ländern hat diese Zusammenarbeit schon lange Tradition. Nun ist es Zeit, dass auch die europäische Ebene in diese Zusammenarbeit einbezogen wird. Nur so kann die Mitwirkung an deutscher Europapolitik im Sinne eines positiven Gestaltungsprozesses gelingen.

84 Wessels/Traguth: Der hauptamtliche Präsident des Europäischen Rates, 2010. 